6-10-2014

\title{
Lead Optimization of Dual Tubulin and Hsp27 Inhibitors
}

\author{
Bo Zhong \\ Cleveland State University \\ Rati Lama \\ Cleveland State University \\ Daniel G. Kulman \\ Cleveland State University \\ Bibo Li Ph.D. \\ Cleveland State University, B.LI37@csuohio.edu \\ Bin Su Ph.D.

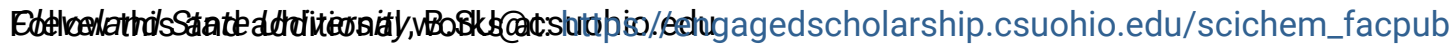 \\ Part of the Medicinal-Pharmaceutical Chemistry Commons
}

How does access to this work benefit you? Let us know!

\section{Publisher's Statement}

NOTICE: this is the author's version of a work that was accepted for publication in European Journal of Medicinal Chemistry. Changes resulting from the publishing process, such as peer review, editing, corrections, structural formatting, and other quality control mechanisms may not be reflected in this document. Changes may have been made to this work since it was submitted for publication. A definitive version was subsequently published in European Journal of Medicinal Chemistry, VOL80, (June 10, 2014) DOI: 10.1016/j.ejmech.2014.04.038

\section{Recommended Citation}

Zhong, B.; Lama, R.; Kulman, D. G.; Li, B.; Su, B. Lead optimization of dual tubulin and Hsp27 inhibitors. Eur. J. Med. Chem. 2014, 80, 243-253.

This Article is brought to you for free and open access by the Chemistry Department at EngagedScholarship@CSU. It has been accepted for inclusion in Chemistry Faculty Publications by an authorized administrator of EngagedScholarship@CSU. For more information, please contact library.es@csuohio.edu. 


\title{
Lead optimization of dual tubulin and Hsp27 inhibitors
}

\author{
Bo Zhong ${ }^{a}$, Rati Lama ${ }^{a}$, Daniel G. Kulman ${ }^{a}$, Bibo Li ${ }^{\text {b.c,**}}$, Bin Su ${ }^{\text {a,c,* }}$ \\ ${ }^{a}$ Department of Chemistry, College of Sciences and Health Professions, Cleveland State University, 2121 Euclid Ave., Cleveland, OH 44115, USA \\ ${ }^{b}$ Department of Biological, Geo. E Env. Sciences, College of Sciences and Health Professions, Cleveland State University, 2121 Euclid Ave., Cleveland, \\ $\mathrm{OH} 44115$, USA \\ ${ }^{c}$ Center for Gene Regulation in Health and Disease, College of Sciences E' Health Professions, Cleveland State University, 2121 Euclid Ave., Cleveland, \\ $\mathrm{OH}$ 44115, USA
}

\section{Introduction}

Stress factors including chemotherapies can enhance the expression of heat shock proteins (Hsp) in mammalian cells [1-3]. Hsp27, a small Hsp with a molecular weight of $27 \mathrm{kDa}$, promotes the survival of mammalian cells exposed to anti-cancer agents [4]. The results of several investigations demonstrate that Hsp27 overexpression leads to the resistance of anti-cancer drugs [3,5]. The cellular protective effects of Hsp27 rely on its molecular chaperone functions against apoptotic signals to eliminate anticancer agents induced programmed cell death [6-8]. Upregulation of Hsp27 leads to an over-active chaperone activity, which contributes to the cancer cell survival under chemotherapeutic agents [1,2]. Increased expression of $\mathrm{Hsp} 27$ has been detected in a number of cancers such as breast cancer, endometrial cancer and leukemia $[1,2,9-13]$, which supports the hypothesis

* Corresponding author. Department of Chemistry, College of Sciences and Health Professions, Cleveland State University, 2121 Euclid Ave., Cleveland, OH 44115, USA

** Corresponding author. Department of Biological, Geo. \& Env. Sciences, College of Sciences and Health Professions, Cleveland State University, 2121 Euclid Ave. Cleveland, $\mathrm{OH} 44115$, USA

E-mail addresses: b.li37@csuohio.edu (B. Li), B.su@csuohio.edu (B. Su). that Hsp27 is a good target of novel cancer therapy. Hsp27 inhibitors can decrease the protective function of the protein in cell apoptotic regulation, and enhance the anti-cancer efficacy of chemotherapy $[5,14,15]$. In fact, several agents diminishing Hsp27 functions exhibited promising anti-tumor activity, and also enhanced the potency of other anti-cancer agents in preclinical models of a variety of tumors expressing high levels of Hsp27 $[9,14,15]$.

Tubulin-containing structures are important for many cellular functions, including chromosome segregation during cell division, intracellular transport, development and maintenance of cell shape, cell motility, and distribution of molecules on cell membrane [16]. Tubulin inhibitors are classical chemotherapeutic agents for cancer treatment. It has been well documented that tubulin inhibitors cause cell cycle arrest and induce apoptosis [17-20]. Several preclinical studies demonstrated that Hsp27 inhibition could potentiate the apoptosis-inducing activity of tubulin inhibitors [21]. Compounds targeting both Hsp27 and tubulin can fuse the two anti-cancer effects into one agent, and create a synergistic activity by itself. These dual tubulin and Hsp27 inhibitors will generate "one stone two birds" effect, and may represent a class of new anti-cancer agents. 
Previously we developed tubulin inhibitor, Hsp27 inhibitor and dual Hsp27 and tubulin inhibitors that exhibited potent anti-cancer activity [22]. In addition, the combination of pure tubulin inhibitor with Hsp27 inhibitor showed a synergistic effect to inhibit cancer cell proliferation [22], suggesting that the compounds with dual molecular targets have advantages over agents with single molecular target. It is desirable to further optimize the dual targeting compounds to generate new analogs with better potency to inhibit both Hsp27 and tubulin, which will lead to more potent anti-cancer agents. In the current study, systematic ligand based optimization resulted in compounds that significantly inhibited breast cancer cell proliferation. Unexpectedly, the Hsp27 inhibitory activity of the compounds was diminished during the lead optimization. The new derivatives became pure tubulin inhibitors. The results revealed several key structural elements critical for Hsp27 inhibition, which provides important structural characteristics for future optimization to improve Hsp27 inhibition.

\section{Results and discussions}

\subsection{Lead optimization and summarization of the structure-activity relationship (SAR)}

Previously, we have shown that structural modification of the lead compound 1 (Fig. 1) by substitution of 2,5-dimethylbenzyl moiety with 2,5-dimethoxybenzyl group dramatically increased the inhibition of cell proliferative activity. Several new derivatives inhibited the proliferation of various cancer cell lines with $\mathrm{IC}_{50}$ values at subnanomolar level [22]. Additionally, replacing methyl sulfonamide group in these compounds with trifluoromethyl sulfonamide group significantly impaired their anti-cancer activity, suggesting that the trifluoromethyl sulfonamide group is not well tolerated. Since no other alternatives to methyl sulfonamide group were investigated in the previous study, we wonder if a bulkier or more hydrophobic group, such as propyl, phenyl, or benzyl at this position, could be tolerated. Therefore, in this study we kept the B moiety as 2,5-dimethoxybenzyl group, and systematically changed the $\mathrm{C}$ moiety to several different sulfonamide groups including ethyl-, propyl-, phenyl-, and benzyl-sulfonamide. The acetamide group, which is smaller than the methyl sulfonamide group and shows different electronic distribution, was also evaluated. Previous studies revealed that the $\mathrm{N}$-methyl group at the $\mathrm{D}$ position is important for the anti-proliferative activity of methyl sulfonamides. Removal of $\mathrm{N}$-methyl group significantly decreased their cell growth inhibitory potency [22]. In this study, we further confirmed this structure-activity relationship on this moiety with this series of compounds. Some analogs were designed as free - NH moiety and some were designed as -Methyl NH moiety. For all the new derivatives, moiety $\mathrm{A}$ was remained as 4-methoxyphenyl, 3,4dimethoxyphenyl, 4-bromophenyl, and 4-iodophenyl group. The previous evaluation of 39 different aryl groups at the A position revealed that these four substituted aryl groups were superior to other ones [22].

All these new compounds were synthesized according to the previous method (Scheme 1) [22]. They were examined for potency and selectivity on the growth inhibition of four different breast cancer cells lines including SKBR-3, MCF-7, MDA-MB-231, and MDA-MB-468. SKBR-3 cell is human epidermal growth factor receptor 2 (HER2) positive and estrogen receptor (ER) negative, while MCF-7 cell is HER2 negative and ER positive. Both MDA-MB-231 and MDA-MB-468 cells are HER2 and ER negative. The results are summarized in Table 1 . The $\mathrm{IC}_{50} \mathrm{~S}$ of cell growth inhibition of the compounds ranges from $0.001 \mu \mathrm{M}$ to $2.44 \mu \mathrm{M}$ for SKBR-3 cells, $0.001 \mu \mathrm{M}$ to $186.0 \mu \mathrm{M}$ for MCF-7 cells, $0.1 \mu \mathrm{M}$ to $48.1 \mu \mathrm{M}$ for MDAMB-231 cells, and $0.008 \mu \mathrm{M}$ to $1.010 \mu \mathrm{M}$ for MDA-MB-468 cells. Most compounds exhibited more potent growth inhibition to SKBR-3 and MDA-MB-468 cells than MCF-7 and MDA-MB-231 cells. For SKBR-3 cells, SAR analysis indicated that the sulfonamide group at the $\mathrm{C}$ moiety of these compounds is critical for the biological activity. The previously synthesized compounds N-methylmethanesulfonamide $\mathbf{2}-\mathbf{5}$ showed potent anti-proliferative activity for SKBR-3 cells with $\mathrm{IC}_{50}$ values about $0.001-0.004 \mu \mathrm{M}$ [22]. However N-methylethanesulfonamides $(\mathbf{6}, \mathbf{1 4}, \mathbf{1 6})$, N-methyl-propylsulfoylamides $(\mathbf{7}, \mathbf{1 1}, \mathbf{1 5}, \mathbf{1 7})$, N-methyl-benzylsulfoylamide (8), and $\mathrm{N}$-methyl-phenylsulfonamides $(\mathbf{9}, \mathbf{1 3})$ showed decreased activity compared to the corresponding N-methylmethanesulfonamide (2-5). Only N-methylethanesulfonamide 10 and $\mathrm{N}$-methylbenzylsulfonamide $\mathbf{1 2}$ maintained the potent SKBR3 cell growth inhibition activity with $\mathrm{IC}_{50} \mathrm{~S}$ of $0.001 \mu \mathrm{M}$ and $0.003 \mu \mathrm{M}$ respectively. The general order of potency of various $\mathrm{N}$ methyl alkyl/arylsulfonamides for inhibition of SKBR-3 cell growth is methanesulfonamides $>$ ethanesulfonamides $>=$ propylsulfonamides $>$ benzylsulfoylamides $>$ phenylsulfonamides. The anti-proliferative activity of the compounds against SKBR-3 cells seems to be inversely proportional to the size of alkyl/aryl sulfonamide at the $\mathrm{C}$ moiety. Previous study showed that removal of the $\mathrm{N}$-methyl group in $\mathrm{N}$-methylmethanesulfonamides significantly impairs their biological activity [22]. Similar phenomenon was observed for most N-methyl alkyl/arylsulfonamides evaluated in the current study. Removal of $\mathrm{N}$-methyl group in $\mathrm{N}$-methylethanesulfonamides $(\mathbf{1 0}, \mathbf{1 4}, \mathbf{1 6}), \mathrm{N}$-methyl-propylsulfonamides (7, 11, 15, 17), N-methyl-benzylsulfonamide $(\mathbf{8}, 12)$, and N-methylphenylsulfonamides $(\mathbf{9}, \mathbf{1 3})$, generated the corresponding ethanesulfonamides $(\mathbf{2 2}, \mathbf{2 6}, \mathbf{2 8})$, propylsulfonamides $(\mathbf{1 9}, \mathbf{2 3}, \mathbf{2 7}, \mathbf{2 9})$, benzylsulfonamide $(\mathbf{2 0}, \mathbf{2 4})$, and phenylsulfonamides $(\mathbf{2 1}, \mathbf{2 5})$ with $2-43$ fold loss in potency. The results confirmed that the free $-\mathrm{NH}$ moiety of these compounds decrease the anti-cancer activity. Free$\mathrm{NH}$ can act as a hydrogen bond donor or acceptor, whereas methylNR can only act as hydrogen bond acceptor. The hydrogen bond formation on this moiety may be critical for the biological activity

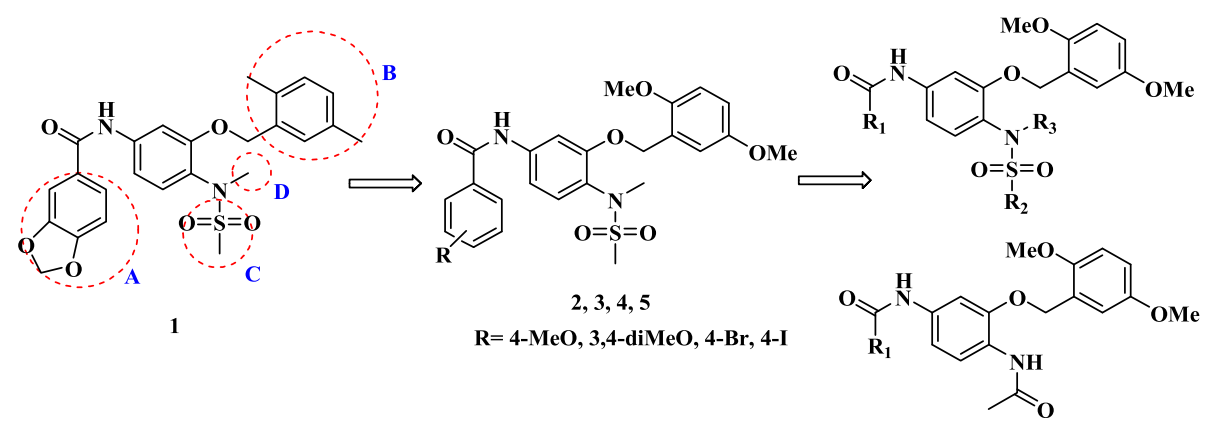

Fig. 1. Ligand based optimization of compound $\mathbf{1}$. 
SAR of alternate A and B moiety methanesulfonamide.<smiles>[R17]C(=O)Nc1ccc(N([R1])S([R2])(=O)=O)c(OCc2cc(OC)ccc2OC)c1</smiles><smiles>[R]C(=O)Nc1ccc(NC(C)=O)c(OCc2cc(OC)ccc2OC)c1</smiles>

\begin{tabular}{|c|c|c|c|c|c|c|c|}
\hline \multirow[t]{2}{*}{ Entry } & \multirow[t]{2}{*}{$\mathrm{R}_{1}(\mathrm{R})$} & \multirow[t]{2}{*}{$\mathrm{R}_{2}$} & \multirow[t]{2}{*}{$\mathrm{R}_{3}$} & \multicolumn{4}{|l|}{$\mathrm{IC}_{50}(\mu \mathrm{M})$} \\
\hline & & & & SKBR-3 & MCF-7 & MDA-MB-231 & MDA-MB-468 \\
\hline 6 & & $\mathrm{CH}_{2} \mathrm{CH}_{3}$ & $\mathrm{CH}_{3}$ & $0.021 \pm 0.004$ & $0.0008 \pm 0.0006$ & $2.03 \pm 1.12$ & $0.014 \pm 0.005$ \\
\hline 7 & & $\mathrm{n}-\mathrm{C}_{3} \mathrm{H}_{7}$ & $\mathrm{CH}_{3}$ & $0.026 \pm 0.02$ & $0.074 \pm 0.042$ & $0.42 \pm 0.29$ & $0.057 \pm 0.021$ \\
\hline 8 & & $\mathrm{PhCH}_{2}$ & $\mathrm{CH}_{3}$ & $0.06 \pm 0.02$ & $0.23 \pm 0.09$ & $14.2 \pm 8.81$ & $0.008 \pm 0.004$ \\
\hline 9 & & $\mathrm{Ph}$ & $\mathrm{CH}_{3}$ & $0.06 \pm 0.03$ & $1.25 \pm 0.59$ & $0.87 \pm 0.51$ & $0.08 \pm 0.02$ \\
\hline 10 & & $\mathrm{CH}_{2} \mathrm{CH}_{3}$ & $\mathrm{CH}_{3}$ & $0.001 \pm 0.0002$ & $0.016 \pm 0.01$ & $0.125 \pm 0.07$ & $0.012 \pm 0.005$ \\
\hline 11 & & $\mathrm{n}-\mathrm{C}_{3} \mathrm{H}_{7}$ & $\mathrm{CH}_{3}$ & $0.007 \pm 0.003$ & $0.14 \pm 0.06$ & $17.9 \pm 12.2$ & $0.008 \pm 0.003$ \\
\hline 12 & & $\mathrm{PhCH}_{2}$ & $\mathrm{CH}_{3}$ & $0.003 \pm 0.001$ & $0.12 \pm 0.06$ & $2.25 \pm 1.51$ & $0.012 \pm 0.005$ \\
\hline 13 & & $\mathrm{Ph}$ & $\mathrm{CH}_{3}$ & $0.05 \pm 0.02$ & $2.88 \pm 1.72$ & $0.8 \pm 0.44$ & $0.05 \pm 0.01$ \\
\hline 14 & & $\mathrm{CH}_{2} \mathrm{CH}_{3}$ & $\mathrm{CH}_{3}$ & $0.056 \pm 0.021$ & $0.097 \pm 0.06$ & $0.1 \pm 0.06$ & $0.088 \pm 0.059$ \\
\hline 15 & & $\mathrm{n}-\mathrm{C}_{3} \mathrm{H}_{7}$ & $\mathrm{CH}_{3}$ & $0.05 \pm 0.02$ & $1.27 \pm 0.69$ & $0.69 \pm 0.41$ & $0.07 \pm 0.02$ \\
\hline 16 & & $\mathrm{CH}_{2} \mathrm{CH}_{3}$ & $\mathrm{CH}_{3}$ & $0.012 \pm 0.005$ & $0.16 \pm 0.1$ & $0.5 \pm 0.31$ & $0.056 \pm 0.022$ \\
\hline 17 & & $\mathrm{n}-\mathrm{C}_{3} \mathrm{H}_{7}$ & $\mathrm{CH}_{3}$ & $0.019 \pm 0.01$ & $0.06 \pm 0.03$ & $21.7 \pm 14.5$ & $0.072 \pm 0.032$ \\
\hline 18 & & $\mathrm{CH}_{2} \mathrm{CH}_{3}$ & $\mathrm{H}$ & $0.019 \pm 0.008$ & $11.9 \pm 6.64$ & $0.16 \pm 0.09$ & $0.048 \pm 0.017$ \\
\hline 19 & & $\mathrm{n}-\mathrm{C}_{3} \mathrm{H}_{7}$ & $\mathrm{H}$ & $0.071 \pm 0.021$ & $0.28 \pm 0.17$ & $4.6 \pm 2.4$ & $0.025 \pm 0.007$ \\
\hline
\end{tabular}




\begin{tabular}{|c|c|c|c|c|c|c|c|}
\hline \multirow[t]{2}{*}{ Entry } & \multirow[t]{2}{*}{$\mathrm{R}_{1}(\mathrm{R})$} & \multirow[t]{2}{*}{$\mathrm{R}_{2}$} & \multirow[t]{2}{*}{$\mathrm{R}_{3}$} & \multicolumn{4}{|l|}{$\mathrm{IC}_{50}(\mu \mathrm{M})$} \\
\hline & & & & SKBR-3 & MCF-7 & MDA-MB-231 & MDA-MB-468 \\
\hline 20 & & $\mathrm{PhCH}_{2}$ & $\mathrm{H}$ & $0.19 \pm 0.09$ & $1.29 \pm 0.7$ & $1.83 \pm 1.01$ & $0.09 \pm 0.04$ \\
\hline 21 & & $\mathrm{Ph}$ & $\mathrm{H}$ & $0.31 \pm 0.11$ & $16.7 \pm 7.44$ & $12.4 \pm 5.9$ & $0.72 \pm 0.23$ \\
\hline 22 & & $\mathrm{CH}_{2} \mathrm{CH}_{3}$ & $\mathrm{H}$ & $0.026 \pm 0.005$ & $0.44 \pm 0.12$ & $19.8 \pm 13.1$ & $0.073 \pm 0.029$ \\
\hline 23 & & $\mathrm{n}-\mathrm{C}_{3} \mathrm{H}_{7}$ & $\mathrm{H}$ & $0.047 \pm 0.023$ & $0.05 \pm 0.03$ & $1.84 \pm 0.73$ & $0.019 \pm 0.007$ \\
\hline 24 & & $\mathrm{PhCH}_{2}$ & $\mathrm{H}$ & $0.12 \pm 0.04$ & $1.69 \pm 0.91$ & $1.78 \pm 0.86$ & $0.11 \pm 0.03$ \\
\hline 25 & & $\mathrm{Ph}$ & $\mathrm{H}$ & $0.34 \pm 0.17$ & $39.6 \pm 21.7$ & $5.74 \pm 3.07$ & $0.18 \pm 0.06$ \\
\hline 26 & & $\mathrm{CH}_{2} \mathrm{CH}_{3}$ & $\mathrm{H}$ & $0.108 \pm 0.048$ & $2.48 \pm 1.55$ & $0.53 \pm 0.29$ & $0.061 \pm 0.019$ \\
\hline 27 & & $\mathrm{n}-\mathrm{C}_{3} \mathrm{H}_{7}$ & $\mathrm{H}$ & $0.096 \pm 0.037$ & $0.29 \pm 0.16$ & $5.66 \pm 2.02$ & $0.45 \pm 0.14$ \\
\hline 28 & & $\mathrm{CH}_{2} \mathrm{CH}_{3}$ & $\mathrm{H}$ & $0.085 \pm 0.031$ & $2.51 \pm 1.5$ & $2.31 \pm 1.29$ & $0.042 \pm 0.014$ \\
\hline 29 & & $\mathrm{n}-\mathrm{C}_{3} \mathrm{H}_{7}$ & $\mathrm{H}$ & $0.074 \pm 0.025$ & $0.36 \pm 0.21$ & $20.6 \pm 11.4$ & $0.24 \pm 0.09$ \\
\hline 30 & & & & $0.38 \pm 0.13$ & $1.17 \pm 0.49$ & $19.0 \pm 6.25$ & $0.25 \pm 0.08$ \\
\hline 31 & & & & $1.24 \pm 0.32$ & $1.81 \pm 0.7$ & $31.7 \pm 8.65$ & $0.99 \pm 0.25$ \\
\hline 32 & & & & $2.44 \pm 1.53$ & $186.0 \pm 99.3$ & $48.1 \pm 23.6$ & $1.01 \pm 0.44$ \\
\hline 33 & & & & $0.11 \pm 0.02$ & $0.31 \pm 0.17$ & $3.65 \pm 1.87$ & $0.29 \pm 0.04$ \\
\hline
\end{tabular}

comparable to that of $\mathrm{N}$-methylmethanesulfonamide $\mathbf{5}$ from the previous study [22] (see Fig. 3).

\subsection{Tubulin docking studies}

In the previous study, lead compound $\mathbf{1}$ was found to be a colchicine site binder on tubulin [23]. We anticipate the new derivatives $\mathbf{1 0}$ and $\mathbf{1 2}$ should also target the colchicine-binding site in tubulin, due to their structural similarity to compound $\mathbf{1}$. To further elucidate the interaction between tubulin and compounds $\mathbf{1 0}$ and 12, we performed a docking study of these two compounds into the colchicine-binding site in tubulin. The results are shown in Fig. 4. Compound $\mathbf{1 0}$ may form three major interactions with tubulin. First, 2-methoxyl group in 2,5-dimethoxybenzyl moiety may form a hydrogen bond with the side chain of Ser 178 . There may be some polar contact between the sulfonamide group and side chains such 

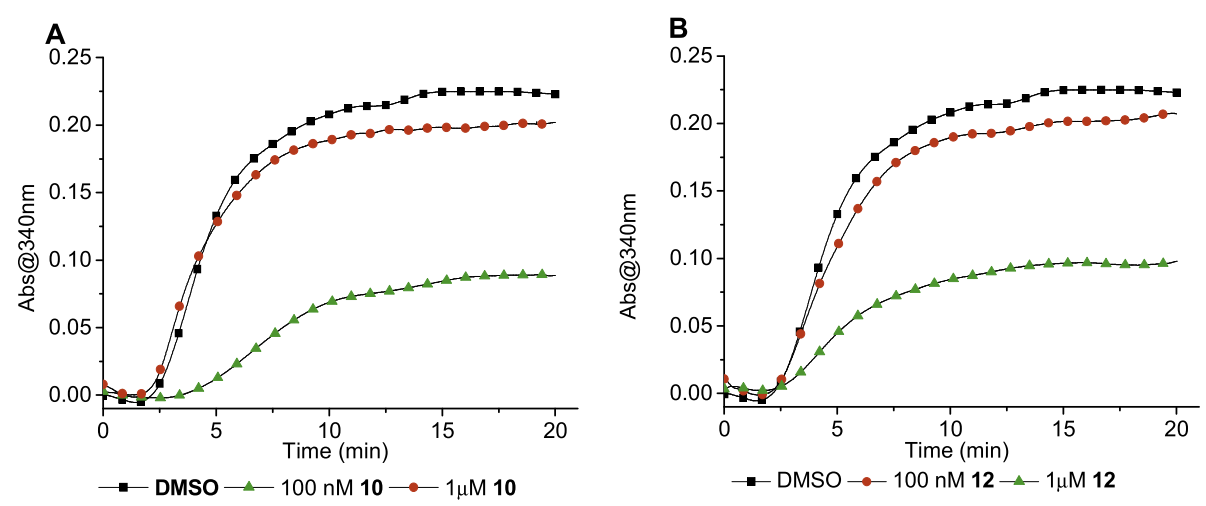

Fig. 2. Tubulin polymerization in the presence of different concentrations of compounds $\mathbf{1 0}$ and $\mathbf{1 2}$.

as Glu 183 and Asn 101. Additionally, the carbonyl group of benzoyl amide moiety may form a hydrogen bond with the main chain of Ala 250. The docking investigation showed that compound $\mathbf{1 0}$ has strong binding affinity to the colchicine-binding site of tubulin. Compared to compound 10, compound 12 showed a different binding mode in the colchicine-binding site with sulfonamide moiety and benzoyl amide moiety switched. The benzyl group in the sulfonamide moiety can extend into a small hydrophobic pocket defined by Tyr 202, Ile 378, Leu 242, and Leu 255. A hydrogen bond may be formed between the carbonyl group of benzoyl amide moiety and the side chain of Asn 258. The 2,5dimethoxybenzyl moiety in compound 12 slightly shifted away from the side chain of Ser 178, and the possible hydrogen bond between them was forfeited. However, a hydrophobic interaction may form between 2,5-dimethoxybenzyl moiety and Lys 352 . The estimated free energies for compound $\mathbf{1 0}$ and $\mathbf{1 2}$ for binding at the colchicine site were determined to be -7.57 and $-7.86 \mathrm{kcal} / \mathrm{mol}$, respectively. Their similar binding energy in the colchicine-binding site may account for their similar inhibition to tubulin polymerization.

\subsection{Hsp27 targeting effect investigation}

Previously, lead compound 5 inhibited both Hsp27 and tubulin [22]. The newly synthesized derivatives $\mathbf{1 0}$ and $\mathbf{1 2}$ inhibited tubulin and showed potent anti-proliferative activity. Whether they also target Hsp27 is still unknown. In order to further elucidate their anti-cancer mechanism, the capabilities of both compounds to modulate the in vitro chaperone function of Hsp27 were evaluated. By monitoring dithiothreitol (DTT)-induced insulin aggregation in the presence of Hsp27, with or without the compounds, their Hsp27 inhibition can be examined. In this in vitro chaperone activity assays, Hsp27 exhibited potent inhibition of DTT-induced insulin aggregation. Previous study showed that the corresponding $\mathrm{N}$-methylmethanesulfonamide $\mathbf{5}$ at $10 \mu \mathrm{M}$ inhibited Hsp27 functions by 27\% [22]. However, both compounds $\mathbf{1 0}$ and $\mathbf{1 2}$ did not show inhibitory activity against Hsp27 chaperone activity at $10 \mu \mathrm{M}$, suggesting Hsp27 targeting effect decreased in the new compounds. Substitution of the methanesulfonamide at the $C$ moiety of compound $\mathbf{5}$ with ethanesulfonamide or benzylsulfonamide is detrimental for its Hsp27 targeting effect. However, this modification did not affect tubulin targeting effects. The results suggest that smaller sulfonamide moiety is preferred for Hsp27 inhibition.

\section{Conclusion}

We synthesized various sulfonamide derivatives and acetamide derivatives based on the previously reported compounds $\mathbf{2}-\mathbf{5}$ [22]. The 2,5-dimethoxybenzyl group, which had been demonstrated to be important for the anti-proliferative activity of these compounds, was maintained for all the new compounds. The methanesulfonamide group at the $\mathrm{C}$ moiety was changed to an acetamide group or a diversity of alkyl/aryl sulfonamide groups. The SAR study revealed that most ethyl-, propyl-, phenyl-, benzylsulfonamides showed weaker cell growth inhibition, compared to the corresponding methanesulfonamides. Only N-methylethanesulfonamide 10 and $\mathrm{N}$-methylbenzylsulfonamide 12 maintained similar potency. Further mechanism investigation indicated that compounds $\mathbf{1 0}$ and $\mathbf{1 2}$ are potent inhibitors of tubulin polymerization. Their tubulin inhibitory activities are comparable to the
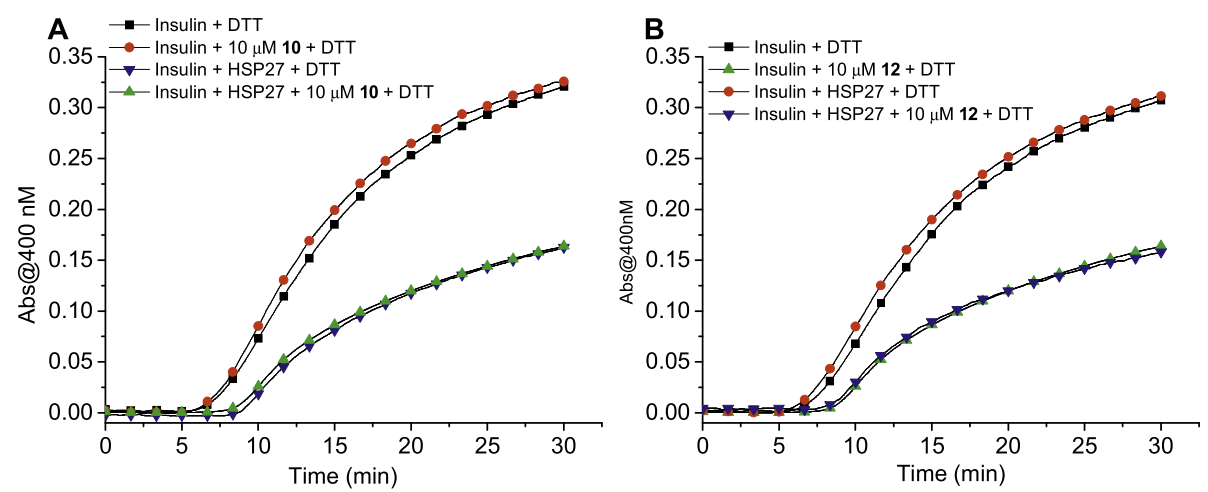

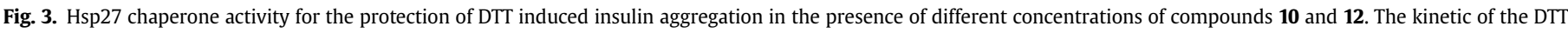
reduction-induced insulin aggregation was monitored in the absence of Hsp27 or in the presence of Hsp27, with or without compounds. 


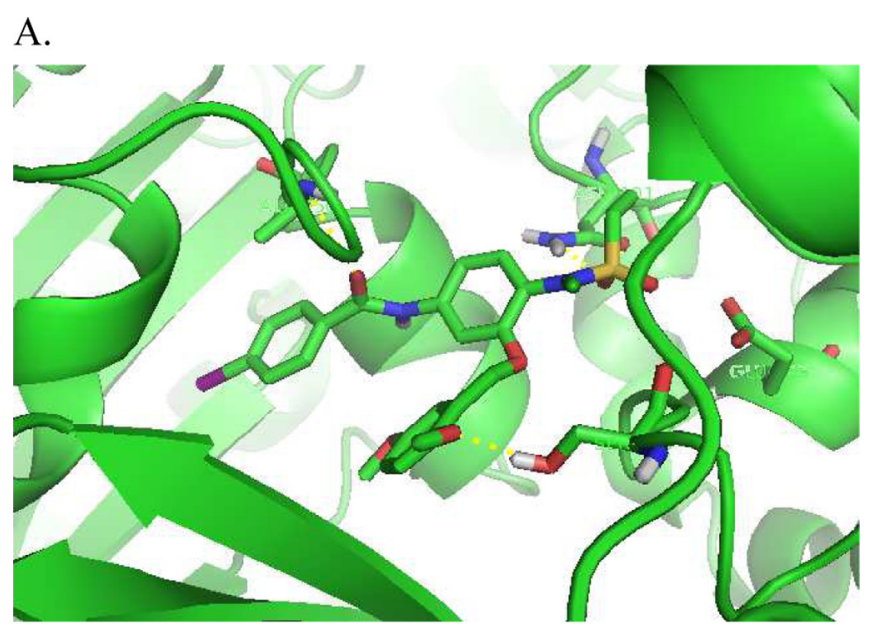

B.

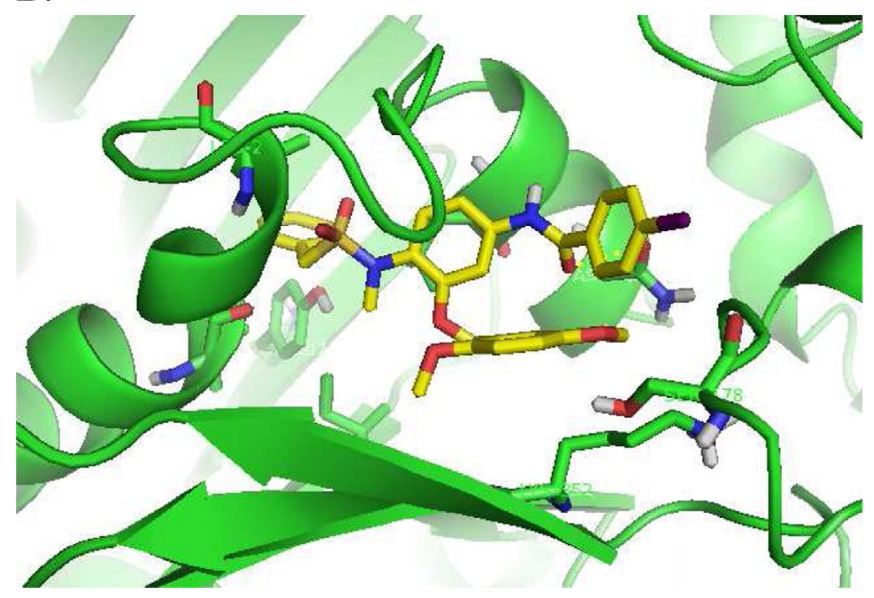

Fig. 4. The molecular docking simulation of the interaction of tubulin with compounds $\mathbf{1 0}$ and 12. Proposed binding mode of compound $\mathbf{1 0}(\mathrm{A})$ and $\mathbf{1 2}$ (B) at the colchicine binding site. The tubulin polypeptide backbones are shown as ribbons. Only important active site amino acids around compounds are shown for clarity.

corresponding lead compound N-methylmethanesulfonamide $\mathbf{5}$. However, both compounds did not show Hsp27 inhibition. The substitution of methanesulfonamide with ethanesulfonamide or benzylsulfonamide significantly impaired the Hsp27 inhibitory effects. The molecular docking simulation suggested that compounds 10 and 12 may adopt different binding modes to be accommodated in the colchicine binding site of tubulin. Future study will focus on discerning the structural fragments that are important for Hsp27 inhibition, and develop new anti-cancer agents with better potency to target both tubulin and Hsp27.

\section{Experimental section}

\subsection{Chemistry}

Chemicals were commercially available and used as received without further purification. Moisture sensitive reactions were carried out under a dry argon atmosphere in flame-dried glassware. Thin-layer chromatography was performed on silica gel TLC plates with fluorescence indicator $254 \mathrm{~nm}$ (Fluka). Flash column chromatography was performed using silica gel $60 \AA$ (BDH, 40-63 $\mu \mathrm{M})$. Mass spectra were obtained on the ABI QStar Electrospray mass spectrometer at Cleveland State University MS facility Center. All the NMR spectra were recorded on a Bruker $400 \mathrm{MHz}\left({ }^{13} \mathrm{C}\right.$ NMR at
$100 \mathrm{MHz}$ ) using DMSO- $d_{6}$ as solvent. Chemical shifts $(\delta)$ for ${ }^{1} \mathrm{H}$ NMR spectra were reported in parts per million to residual solvent protons. The IR spectra were obtained on a Bruker ALPHA FT-IR spectrometer with ATR module.

All the compounds were prepared according to the previously published procedures [22].

4.1.1. $\mathrm{N}$-[3-(2,5-dimethoxy-benzyloxy)-4-(ethanesulfonyl-methylamino)-phenyl]-4-methoxybenzamide (6)

${ }^{1} \mathrm{H}$ NMR $\delta 10.200(1 \mathrm{H}, \mathrm{s}), 7.972(2 \mathrm{H}, \mathrm{d}, J=8.8 \mathrm{~Hz}), 7.732(1 \mathrm{H}, \mathrm{d}$, $J=2.4 \mathrm{~Hz}), 7.415(1 \mathrm{H}, \mathrm{dd}, J=2.4,8.4 \mathrm{~Hz}), 7.250(1 \mathrm{H}, \mathrm{d}, J=8.8 \mathrm{~Hz})$, $7.156(1 \mathrm{H}, \mathrm{d}, J=2.8 \mathrm{~Hz}), 7.081(2 \mathrm{H}, \mathrm{d}, J=9.2 \mathrm{~Hz}), 7.001(1 \mathrm{H}, \mathrm{d}$, $J=9.2 \mathrm{~Hz}), 6.905(1 \mathrm{H}, \mathrm{dd}, J=2.8,8.8 \mathrm{~Hz}), 5.083(2 \mathrm{H}, \mathrm{s}), 3.848(3 \mathrm{H}, \mathrm{s})$, $3.785(3 \mathrm{H}, \mathrm{s}), 3.717(3 \mathrm{H}, \mathrm{s}), 3.136(3 \mathrm{H}, \mathrm{s}), 3.056(2 \mathrm{H}, \mathrm{q}, J=7.6 \mathrm{~Hz})$, $1.114(3 \mathrm{H}, \mathrm{t}, J=7.6 \mathrm{~Hz}) ;{ }^{13} \mathrm{C}$ NMR $\delta 165.43,162.52,155.62,153.57$, $151.37,140.94,131.79,130.10,127.20,125.53,124.70,115.45,114.39$, 114.12, 112.68, 112.28, 105.58, 65.45, 56.27, 55.93, 55.88, 46.09, 38.36, 8.34; ESI-MS calculated for $\mathrm{C}_{26} \mathrm{H}_{31} \mathrm{~N}_{2} \mathrm{O}_{7} \mathrm{~S}[\mathrm{M}+\mathrm{H}]^{+}$515.19, found: 514.98 .

4.1.2. $\mathrm{N}$-[3-(2,5-dimethoxy-benzyloxy)-4-(propanesulfonyl-methylamino)-phenyl]-4-methoxybenzamide (7)

${ }^{1} \mathrm{H}$ NMR $\delta 10.200(1 \mathrm{H}, \mathrm{s}), 7.972(2 \mathrm{H}, \mathrm{d}, J=9.2 \mathrm{~Hz}), 7.732(1 \mathrm{H}, \mathrm{d}$, $J=2 \mathrm{~Hz}), 7.416(1 \mathrm{H}, \mathrm{dd}, J=2,8.4 \mathrm{~Hz}), 7.246(1 \mathrm{H}, \mathrm{d}, J=8.4 \mathrm{~Hz}), 7.148$ $(1 \mathrm{H}, \mathrm{d}, J=3.2 \mathrm{~Hz}), 7.081(2 \mathrm{H}, \mathrm{d}, J=9.2 \mathrm{~Hz}), 7.006(1 \mathrm{H}, \mathrm{d}, J=9.2 \mathrm{~Hz})$, $6.908(1 \mathrm{H}, \mathrm{dd}, J=3.2,9.2 \mathrm{~Hz}), 5.080(2 \mathrm{H}, \mathrm{s}), 3.847(3 \mathrm{H}, \mathrm{s}), 3.787(3 \mathrm{H}$, s), $3.715(3 \mathrm{H}, \mathrm{s}), 3.134(3 \mathrm{H}, \mathrm{s}), 2.984(2 \mathrm{H}, \mathrm{m}), 1.624(2 \mathrm{H}, \mathrm{m}), 0.812$ $(3 \mathrm{H}, \mathrm{t}, J=7.2 \mathrm{~Hz}) ;{ }^{13} \mathrm{C}$ NMR $\delta 165.43,162.52,155.61,153.59,151.37$, 140.91, 131.79, 130.10, 127.20, 125.55, 124.77, 115.51, 114.31, 114.12, 112.69, 112.36,105.63, 65.45, 56.32, 55.93, 55.87, 53.09, 38.15, 17.21, 13.13; ESI-MS calculated for $\mathrm{C}_{27} \mathrm{H}_{33} \mathrm{~N}_{2} \mathrm{O}_{7} \mathrm{~S}[\mathrm{M}+\mathrm{H}]^{+} 529.20$, found: 529.01.

4.1.3. $\mathrm{N}$-[3-(2,5-dimethoxy-benzyloxy)-4-(benzylsulfonyl-methylamino)-phenyl]-4-methoxybenzamide (8)

${ }^{1} \mathrm{H}$ NMR $\delta 10.191(1 \mathrm{H}, \mathrm{s}), 7.971(2 \mathrm{H}, \mathrm{d}, J=8.8 \mathrm{~Hz}), 7.722(1 \mathrm{H}, \mathrm{d}$, $J=2.4 \mathrm{~Hz}), 7.354(6 \mathrm{H}, \mathrm{m}), 7.204(1 \mathrm{H}, \mathrm{d}, J=2.8 \mathrm{~Hz}), 7.081(2 \mathrm{H}, \mathrm{d}$, $J=9.2 \mathrm{~Hz}), 6.991(1 \mathrm{H}, \mathrm{d}, J=8.8 \mathrm{~Hz}), 6.961(1 \mathrm{H}, \mathrm{d}, J=9.2 \mathrm{~Hz}), 6.867$ $(1 \mathrm{H}, \mathrm{dd}, J=2.8,8.8 \mathrm{~Hz}), 5.115(2 \mathrm{H}, \mathrm{s}), 4.415(2 \mathrm{H}, \mathrm{s}), 3.848(3 \mathrm{H}, \mathrm{s})$, $3.741(3 \mathrm{H}, \mathrm{s}), 3.680(3 \mathrm{H}, \mathrm{s}), 3.074(3 \mathrm{H}, \mathrm{s}) ;{ }^{13} \mathrm{C}$ NMR $\delta 165.43,162.53$, 155.70, 153.64, 151.21, 140.89, 131.40, 131.34, 130.38, 130.10, 128.74, $128.53,127.20,125.61,125.03,115.08,114.43,114.12,112.63,112.28$, $105.59,65.45,57.21,56.29,55.93,55.84,38.46$; ESI-MS calculated for $\mathrm{C}_{31} \mathrm{H}_{33} \mathrm{~N}_{2} \mathrm{O}_{7} \mathrm{~S}[\mathrm{M}+\mathrm{H}]^{+}$577.20, found 576.98.

\subsection{4. $\mathrm{N}$-[3-(2,5-dimethoxy-benzyloxy)-4-(phenylsulfonyl-methyl-} amino)-phenyl]-4-methoxybenzamide (9)

${ }^{1} \mathrm{H}$ NMR $\delta 10.192(1 \mathrm{H}, \mathrm{s}), 7.958(2 \mathrm{H}, \mathrm{d}, J=8.8 \mathrm{~Hz}), 7.624(3 \mathrm{H}, \mathrm{m})$, $7.539(1 \mathrm{H}, \mathrm{m}), 7.441(3 \mathrm{H}, \mathrm{m}), 7.074(3 \mathrm{H}, \mathrm{m}), 6.946(1 \mathrm{H}, \mathrm{d}, J=8.8 \mathrm{~Hz})$, $6.834(2 \mathrm{H}, \mathrm{m}), 4.802(2 \mathrm{H}, \mathrm{s}), 3.845(3 \mathrm{H}, \mathrm{s}), 3.769(3 \mathrm{H}, \mathrm{s}), 3.714(3 \mathrm{H}$, s), $3.146(3 \mathrm{H}, \mathrm{s}) ;{ }^{13} \mathrm{C}$ NMR $\delta 165.46,162.52,155.65,153.54,150.67$, $141.07,139.40,133.01,131.05,130.09,129.33,127.45,127.20,125.61$, 124.38, 114.31, 114.11, 113.67, 112.51, 112.01, 105.66, 65.00, 56.21, 55.92, 55.84, 38.24; ESI-MS calculated for $\mathrm{C}_{30} \mathrm{H}_{31} \mathrm{~N}_{2} \mathrm{O}_{7} \mathrm{~S}[\mathrm{M}+\mathrm{H}]^{+}$ 563.19, found: 562.96 .

4.1.5. N-[3-(2,5-dimethoxy-benzyloxy)- 4-(ethanesulfonyl-methylamino)-phenyl]-4-iodobenzamide (10)

IR $\nu=1672(\mathrm{C}=0)$; ${ }^{1} \mathrm{H}$ NMR $\delta 10.398(1 \mathrm{H}, \mathrm{s}), 7.943(2 \mathrm{H}, \mathrm{d}$, $J=8.4 \mathrm{~Hz}), 7.754(2 \mathrm{H}, \mathrm{d}, J=8.4 \mathrm{~Hz}), 7.703(1 \mathrm{H}, \mathrm{d}, J=2 \mathrm{~Hz}), 7.410$ $(1 \mathrm{H}, \mathrm{dd}, J=2,8.8 \mathrm{~Hz}), 7.267(1 \mathrm{H}, \mathrm{d}, J=8.8 \mathrm{~Hz}), 7.148(1 \mathrm{H}, \mathrm{d}$, $J=3.2 \mathrm{~Hz}), 6.998(1 \mathrm{H}, \mathrm{d}, J=9.2 \mathrm{~Hz}), 6.904(1 \mathrm{H}, \mathrm{dd}, J=3.2,8.8 \mathrm{~Hz})$, $5.083(2 \mathrm{H}, \mathrm{s}), 3.781(3 \mathrm{H}, \mathrm{s}), 3.714(3 \mathrm{H}, \mathrm{s}), 3.135(3 \mathrm{H}, \mathrm{s}), 3.059(2 \mathrm{H}, \mathrm{q}$, $J=7.2 \mathrm{~Hz}), 1.114(3 \mathrm{H}, \mathrm{t}, J=7.2 \mathrm{~Hz}) ;{ }^{13} \mathrm{C}$ NMR $\delta 165.63,155.64,153.49$, $151.40,140.32,137.83,134.48,131.82,130.03,125.39,125.20,115.50$, 
$114.46,112.96,112.34,105.83,99.80,65.49,56.27,55.86,46.15$, 38.30, 8.26; ESI-MS calculated for $\mathrm{C}_{25} \mathrm{H}_{28} \mathrm{IN}_{2} \mathrm{O}_{6} \mathrm{~S}[\mathrm{M}+\mathrm{H}]^{+} 611.07$, found: 610.88 .

4.1.6. $\mathrm{N}$-[3-(2,5-dimethoxy-benzyloxy)- 4-(propanesulfonyl-

methyl-amino)-phenyl]-4-iodobenzamide (11)

${ }^{1} \mathrm{H}$ NMR $\delta 10.399(1 \mathrm{H}, \mathrm{s}), 7.944(2 \mathrm{H}, \mathrm{d}, J=8.8 \mathrm{~Hz}), 7.756(2 \mathrm{H}, \mathrm{d}$, $J=8.8 \mathrm{~Hz}), 7.704(1 \mathrm{H}, \mathrm{d}, J=2.4 \mathrm{~Hz}), 7.412(1 \mathrm{H}, \mathrm{dd}, J=2,8.4 \mathrm{~Hz})$, $7.264(1 \mathrm{H}, \mathrm{d}, J=8.8 \mathrm{~Hz}), 7.140(1 \mathrm{H}, \mathrm{d}, 3.2 \mathrm{~Hz}), 7.004(1 \mathrm{H}, \mathrm{d}$, $J=8.8 \mathrm{~Hz}), 6.907(1 \mathrm{H}, \mathrm{dd}, J=2.8,8.8 \mathrm{~Hz}), 5.080(2 \mathrm{H}, \mathrm{s}), 3.784(3 \mathrm{H}, \mathrm{s})$, $3.712(3 \mathrm{H}, \mathrm{s}), 3.133(3 \mathrm{H}, \mathrm{s}), 2.985(2 \mathrm{H}, \mathrm{m}), 1.623(2 \mathrm{H}, \mathrm{m}), 0.812(3 \mathrm{H}, \mathrm{t}$, $J=7.6 \mathrm{~Hz}) ;{ }^{13} \mathrm{C}$ NMR $\delta 165.35,155.64,153.59,151.36,140.47,137.78$, $134.54,131.86,130.07,125.50,125.18,115.50,114.33,112.84,112.38$, 105.78, 99.94, 65.49, 56.33, 55.88, 53.11, 38.14, 17.21, 13.13; ESI-MS calculated for $\mathrm{C}_{26} \mathrm{H}_{30} \mathrm{IN}_{2} \mathrm{O}_{6} \mathrm{~S}[\mathrm{M}+\mathrm{H}]^{+} 625.09$, found 624.87 .

\subsection{7. $\mathrm{N}$-[3-(2,5-dimethoxy-benzyloxy)-4-(benzylsulfonyl-methyl-} amino)-phenyl]-4-iodobenzamide (12)

IR $\nu=1645(\mathrm{C}=0)$; ${ }^{1} \mathrm{H}$ NMR $\delta 10.391(1 \mathrm{H}, \mathrm{s}), 7.944(2 \mathrm{H}, \mathrm{d}$, $J=8.4 \mathrm{~Hz}), 7.754(2 \mathrm{H}, \mathrm{d}, J=8.4 \mathrm{~Hz}), 7.692(1 \mathrm{H}, \mathrm{d}, J=2.4 \mathrm{~Hz}), 7.353$ $(6 \mathrm{H}, \mathrm{m}), 7.196(1 \mathrm{H}, \mathrm{d}, J=3.2 \mathrm{~Hz}), 7.006(1 \mathrm{H}, \mathrm{d}, J=8.4 \mathrm{~Hz}), 6.959(1 \mathrm{H}$, $\mathrm{d}, J=9.2 \mathrm{~Hz}), 6.866(1 \mathrm{H}, \mathrm{dd}, J=2.8,8.8 \mathrm{~Hz}), 5.114(2 \mathrm{H}, \mathrm{s}), 4.418(2 \mathrm{H}$, s), $3.738(3 \mathrm{H}, \mathrm{s}), 3.677(3 \mathrm{H}, \mathrm{s}), 3.072(3 \mathrm{H}, \mathrm{s}) ;{ }^{13} \mathrm{C}$ NMR $\delta 165.35$, $155.72,153.64,151.20,140.45,137.79,134.54,131.40,130.36,130.07$, 128.74, 128.54, 125.56, 125.43, 115.05, 114.43, 112.77, 112.29, 105.73, $99.95,65.48,57.22,56.30,55.84,38.45$; ESI-MS calculated for $\mathrm{C}_{30} \mathrm{H}_{30} \mathrm{IN}_{2} \mathrm{O}_{6} \mathrm{~S}[\mathrm{M}+\mathrm{H}]^{+}$673.09, found 672.84.

\subsection{8. $\mathrm{N}$-[3-(2,5-dimethoxy-benzyloxy)-4-(phenylsulfonyl-methyl-} amino)-phenyl]-4-iodobenzamide (13)

${ }^{1} \mathrm{H}$ NMR $\delta 10.390(1 \mathrm{H}, \mathrm{s}), 7.937(2 \mathrm{H}, \mathrm{d}, J=8.4 \mathrm{~Hz}), 7.741(2 \mathrm{H}, \mathrm{d}$, $J=8.4 \mathrm{~Hz}), 7.619(3 \mathrm{H}, \mathrm{m}), 7.541(1 \mathrm{H}, \mathrm{m}), 7.431(3 \mathrm{H}, \mathrm{m}), 7.094(1 \mathrm{H}, \mathrm{d}$, $J=8.8 \mathrm{~Hz}), 6.945(1 \mathrm{H}, \mathrm{d}, J=8.8 \mathrm{~Hz}), 6.833(2 \mathrm{H}, \mathrm{m}), 4.801(2 \mathrm{H}, \mathrm{s})$, $3.765(3 \mathrm{H}, \mathrm{s}), 3.712(3 \mathrm{H}, \mathrm{s}), 3.146(3 \mathrm{H}, \mathrm{s}) ;{ }^{13} \mathrm{C}$ NMR $\delta 165.38,155.67$, 153.53, 150.66, 140.64, 139.36, 137.77, 134.54, 133.04, 131.15, 130.07, 129.35, 127.45, 125.56, 124.77, 114.31, 113.69, 112.64, 112.03, 105.79, $99.95, \quad 65.05, \quad 56.22, \quad 55.85, \quad 38.22$; ESI-MS calculated for $\mathrm{C}_{29} \mathrm{H}_{28} \mathrm{IN}_{2} \mathrm{O}_{6} \mathrm{~S}[\mathrm{M}+\mathrm{H}]^{+}$659.07, found: 658.86 .

\subsection{9. $N$-[3-(2,5-dimethoxy-benzyloxy)-4-(ethanesulfonyl-methyl-} amino)-phenyl]-3,4-dimethoxybenzamide (14)

${ }^{1} \mathrm{H}$ NMR $\delta 10.191(1 \mathrm{H}, \mathrm{s}), 7.736(1 \mathrm{H}, \mathrm{d}, J=2.4 \mathrm{~Hz}), 7.643(1 \mathrm{H}, \mathrm{dd}$, $J=2.4,8.4 \mathrm{~Hz}), 7.534(1 \mathrm{H}, \mathrm{d}, J=2 \mathrm{~Hz}), 7.398(1 \mathrm{H}, \mathrm{dd}, J=2,8.4 \mathrm{~Hz})$, $7.259(1 \mathrm{H}, \mathrm{d}, J=8.4 \mathrm{~Hz}), 7.159(1 \mathrm{H}, \mathrm{d}, J=3.2 \mathrm{~Hz}), 7.104(1 \mathrm{H}, \mathrm{d}$, $J=8.4 \mathrm{~Hz}), 7.002(1 \mathrm{H}, \mathrm{d}, J=9.2 \mathrm{~Hz}), 6.907(1 \mathrm{H}, \mathrm{dd}, J=2.8,8.8 \mathrm{~Hz})$, $5.086(2 \mathrm{H}, \mathrm{s}), 3.852(3 \mathrm{H}, \mathrm{s}), 3.847(3 \mathrm{H}, \mathrm{s}), 3.785(3 \mathrm{H}, \mathrm{s}), 3.719(3 \mathrm{H}, \mathrm{s})$, $3.138(3 \mathrm{H}, \mathrm{s}), 3.059(2 \mathrm{H}, \mathrm{q}, J=7.6 \mathrm{~Hz}), 1.115(3 \mathrm{H}, \mathrm{t}, J=7.6 \mathrm{~Hz}) ;{ }^{3} \mathrm{C}$ NMR $\delta 165.47,155.63,153.58,152.32,151.38,148.87,140.88,131.79$, 127.24, 125.53, 124.77, 121.54, 115.47, 114.41, 112.80, 112.29, 111.69, 111.42, 105.69, 65.47, 56.19, 56.16, 55.88, 46.10, 38.36, 8.34; ESI-MS calculated for $\mathrm{C}_{27} \mathrm{H}_{33} \mathrm{~N}_{2} \mathrm{O}_{8} \mathrm{~S}[\mathrm{M}+\mathrm{H}]^{+} 545.20$, found: 544.98 .

4.1.10. N-[3-(2,5-dimethoxy-benzyloxy)-4-(propanesulfonylmethyl-amino)-phenyl]-3,4-dimethoxybenzamide (15)

${ }^{1} \mathrm{H}$ NMR $\delta 10.192(1 \mathrm{H}, \mathrm{s}), 7.735(1 \mathrm{H}, \mathrm{d}, J=2 \mathrm{~Hz}), 7.644(1 \mathrm{H}, \mathrm{dd}$, $J=2,8.4 \mathrm{~Hz}), 7.534(1 \mathrm{H}, \mathrm{d}, J=2 \mathrm{~Hz}), 7.398(1 \mathrm{H}, \mathrm{dd}, J=2,8.4 \mathrm{~Hz})$, $7.256(1 \mathrm{H}, \mathrm{d}, J=8.8 \mathrm{~Hz}), 7.150(1 \mathrm{H}, \mathrm{d}, J=2.8 \mathrm{~Hz}), 7.104(1 \mathrm{H}, \mathrm{d}$, $J=8.4 \mathrm{~Hz}), 7.007(1 \mathrm{H}, \mathrm{d}, 8.8 \mathrm{~Hz}), 6.910(1 \mathrm{H}, \mathrm{dd}, J=3.2,8.8 \mathrm{~Hz}), 5.083$ $(1 \mathrm{H}, \mathrm{s}), 3.582(3 \mathrm{H}, \mathrm{s}), 3.847(3 \mathrm{H}, \mathrm{s}), 3.787(3 \mathrm{H}, \mathrm{s}), 3.717(3 \mathrm{H}, \mathrm{s}), 3.136$ (3H, s), $2.979(2 \mathrm{H}, \mathrm{m}), 1.623(2 \mathrm{H}, \mathrm{m}), 0.811(3 \mathrm{H}, \mathrm{t}, J=7.2 \mathrm{~Hz}) ;{ }^{13} \mathrm{C}$ NMR $\delta 165.46,155.62,153.60,152.31,151.37,148.87,140.85,131.77$, $127.23,125.54,124.88,121.53,115.53,114.34,112.80,112.39,111.69$, 111.41, 105.73, 65.47, 56.33, 56.20, 56.10, 55.88, 53.10, 38.15, 17.21, 13.13; ESI-MS calculated for $\mathrm{C}_{28} \mathrm{H}_{35} \mathrm{~N}_{2} \mathrm{O}_{8} \mathrm{~S}[\mathrm{M}+\mathrm{H}]^{+} 559.20$, found: 559.02 .
4.1.11. $N$-[3-(2,5-dimethoxy-benzyloxy)- 4-(ethanesulfonyl-methylamino)-phenyl]-4-bromobenzamide (16)

${ }^{1} \mathrm{H}$ NMR $\delta 10.422(1 \mathrm{H}, \mathrm{s}), 7.921(2 \mathrm{H}, \mathrm{d}, J=8.8 \mathrm{~Hz}), 7.773(2 \mathrm{H}, \mathrm{d}$, $J=8.8 \mathrm{~Hz}), 7.706(1 \mathrm{H}, \mathrm{d}, J=2 \mathrm{~Hz}), 7.412(1 \mathrm{H}, \mathrm{dd}, J=2.4,8.8 \mathrm{~Hz})$, $7.272(1 \mathrm{H}, \mathrm{d}, J=8.8 \mathrm{~Hz}), 7.149(1 \mathrm{H}, \mathrm{d}, J=3.2 \mathrm{~Hz}), 7.000(1 \mathrm{H}, \mathrm{d}$, $J=9.2 \mathrm{~Hz}), 6.905(1 \mathrm{H}, \mathrm{dd}, J=3.2,9.2 \mathrm{~Hz}), 5.086(2 \mathrm{H}, \mathrm{s}), 3.782(3 \mathrm{H}, \mathrm{s})$, $3.714(3 \mathrm{H}, \mathrm{s}), 3.137(3 \mathrm{H}, \mathrm{s}), 3.062(2 \mathrm{H}, \mathrm{q}, J=7.6 \mathrm{~Hz}), 1.116(3 \mathrm{H}, \mathrm{t}$, $J=7.6 \mathrm{~Hz}) ;{ }^{13} \mathrm{C}$ NMR $\delta 165.09,155.65,153.58,151.37,140.49,134.24$, $131.92,130.27,126.00,125.49,125.14,115.43,114.40,112.83,112.29$, 105.74, 65.49, 56.28, 55.88, 46.11, 38.34, 8.34; ESI-MS calculated for $\mathrm{C}_{25} \mathrm{H}_{28} \mathrm{BrN}_{2} \mathrm{O}_{6} \mathrm{~S}[\mathrm{M}+\mathrm{H}]^{+}$563.09, found: 562.90 .

\subsubsection{N-[3-(2,5-dimethoxy-benzyloxy)- 4-(propanesulfonyl-} methyl-amino)-phenyl]-4-bromobenzamide (17)

${ }^{1} \mathrm{H}$ NMR $\delta 10.421(1 \mathrm{H}, \mathrm{s}), 7.923(2 \mathrm{H}, \mathrm{d}, J=8.8 \mathrm{~Hz}), 7.773(2 \mathrm{H}, \mathrm{d}$, $J=8.8 \mathrm{~Hz}), 7.708(1 \mathrm{H}, \mathrm{d}, J=1.6 \mathrm{~Hz}), 7.414(1 \mathrm{H}, \mathrm{dd}, J=2,8.8 \mathrm{~Hz})$, $7.270(1 \mathrm{H}, \mathrm{d}, J=8.4 \mathrm{~Hz}), 7.142(1 \mathrm{H}, \mathrm{d}, J=3.2 \mathrm{~Hz}), 7.005(1 \mathrm{H}, \mathrm{d}$, $J=8.8 \mathrm{~Hz}), 6.908(1 \mathrm{H}, \mathrm{dd}, J=2.8,8.8 \mathrm{~Hz}), 5.084(2 \mathrm{H}, \mathrm{s}), 3.785(3 \mathrm{H}, \mathrm{s})$, $3.714(3 \mathrm{H}, \mathrm{s}), 3.136(3 \mathrm{H}, \mathrm{s}), 2.987(2 \mathrm{H}, \mathrm{m}), 1.626(2 \mathrm{H}, \mathrm{m}), 0.814(3 \mathrm{H}, \mathrm{t}$, $J=7.2 \mathrm{~Hz}) ;{ }^{13} \mathrm{C}$ NMR $\delta 165.09,155.64,153.59,151.36,140.46,134.24$, $131.92,130.27,126.00,125.50,125.21,115.50,114.33,112.85,112.38$, $105.79,65.49,56.33,55.87,53.11,38.13,17.21,13.13$; ESI-MS calculated for $\mathrm{C}_{26} \mathrm{H}_{30} \mathrm{BrN}_{2} \mathrm{O}_{6} \mathrm{~S}[\mathrm{M}+\mathrm{H}]^{+}$577.10, found 576.92 .

4.1.13. N-[3-(2,5-dimethoxy-benzyloxy)-4-(ethanesulfonyl-amino)phenyl]-4-methoxybenzamide (18)

${ }^{1} \mathrm{H}$ NMR $\delta 10.136(1 \mathrm{H}, \mathrm{s}), 8.916(1 \mathrm{H}, \mathrm{s}), 7.959(2 \mathrm{H}, \mathrm{d}, J=8.8 \mathrm{~Hz})$, $7.680(1 \mathrm{H}, \mathrm{d}, J=2 \mathrm{~Hz}), 7.384(1 \mathrm{H}, \mathrm{dd}, J=2,8.4 \mathrm{~Hz}), 7.256(1 \mathrm{H}, \mathrm{d}$, $J=3.2 \mathrm{~Hz}), 7.215(1 \mathrm{H}, \mathrm{d}, J=8.4 \mathrm{~Hz}), 7.069(2 \mathrm{H}, \mathrm{d}, J=8.8 \mathrm{~Hz}), 6.984$ $(1 \mathrm{H}, \mathrm{d}, J=8.8 \mathrm{~Hz}), 6.887(1 \mathrm{H}, \mathrm{dd}, J=3.2,8.8 \mathrm{~Hz}), 5.061(2 \mathrm{H}, \mathrm{s}), 3.842$ (3H, s), $3.792(3 \mathrm{H}, \mathrm{s}), 3.724(3 \mathrm{H}, \mathrm{s}), 2.956(2 \mathrm{H}, \mathrm{q}, J=7.2 \mathrm{~Hz}), 1.128$ $(3 \mathrm{H}, \mathrm{t}, J=7.2 \mathrm{~Hz}) ;{ }^{13} \mathrm{C}$ NMR $\delta 165.31,162.44,153.65,152.90,151.16$, 139.10, 130.04, 128.08, 127.30, 125.72, 121.26, 115.58, 114.09, 113.96, $112.84,112.09,105.63,65.49,56.29,55.93,55.91,46.75,8.46$; ESIMS calculated for $\mathrm{C}_{25} \mathrm{H}_{29} \mathrm{~N}_{2} \mathrm{O}_{7} \mathrm{~S}[\mathrm{M}+\mathrm{H}]^{+}$: 501.17 , found: 500.96 .

4.1.14. N-[3-(2,5-dimethoxy-benzyloxy)-4-(propanesulfonylamino)-phenyl]-4-methoxybenzamide (19)

${ }^{1} \mathrm{H}$ NMR $\delta 10.137(1 \mathrm{H}, \mathrm{s}), 8.941(1 \mathrm{H}, \mathrm{s}), 7.960(2 \mathrm{H}, \mathrm{d}, J=9.2 \mathrm{~Hz})$, $7.678(1 \mathrm{H}, \mathrm{d}, J=2 \mathrm{~Hz}), 7.390(1 \mathrm{H}, \mathrm{dd}, J=2,8.8 \mathrm{~Hz}), 7.270(1 \mathrm{H}, \mathrm{d}$, $J=3.2 \mathrm{~Hz}), 7.213(1 \mathrm{H}, \mathrm{d}, J=8.8 \mathrm{~Hz}), 7.070(2 \mathrm{H}, \mathrm{d}, J=9.2 \mathrm{~Hz}), 6.986$ $91 \mathrm{H}, \mathrm{d}, J=9.2 \mathrm{~Hz}), 6.886(1 \mathrm{H}, \mathrm{dd}, J=3.2,9.2 \mathrm{~Hz}), 5.068(2 \mathrm{H}, \mathrm{s}), 3.843$ $(3 \mathrm{H}, \mathrm{s}), 3.797(3 \mathrm{H}, \mathrm{s}), 3.728(3 \mathrm{H}, \mathrm{s}), 2.918(2 \mathrm{H}, \mathrm{m}), 1.654(2 \mathrm{H}, \mathrm{m})$, $0.790(3 \mathrm{H}, \mathrm{t}, J=7.2 \mathrm{~Hz}) ;{ }^{13} \mathrm{C}$ NMR $\delta 165.31,162.44,153.70,152.82$, 151.07, 139.04, 130.04, 127.96, 127.30, 125.80, 121.34, 115.47, 114.09, $113.82,112.88,112.17,105.70,65.42,56.37,55.95,55.92,54.12,17.33$, 13.01; ESI-MS calculated for $\mathrm{C}_{26} \mathrm{H}_{31} \mathrm{~N}_{2} \mathrm{O}_{7} \mathrm{~S}[\mathrm{M}+\mathrm{H}]^{+}$515.19, found: 514.99 .

4.1.15. N-[3-(2,5-dimethoxy-benzyloxy)-4-(benzylsulfonyl-amino)phenyl]-4-methoxybenzamide (20)

${ }^{1} \mathrm{H}$ NMR $\delta 10.149(1 \mathrm{H}, \mathrm{s}), 8.993(1 \mathrm{H}, \mathrm{s}), 7.970(2 \mathrm{H}, \mathrm{d}, J=8.8 \mathrm{~Hz})$, $7.709(1 \mathrm{H}, \mathrm{d}, J=2 \mathrm{~Hz}), 7.384(1 \mathrm{H}, \mathrm{dd}, J=2,8.4 \mathrm{~Hz}), 7.304(6 \mathrm{H}, \mathrm{m})$, $7.150(1 \mathrm{H}, \mathrm{d}, J=8.8 \mathrm{~Hz}), 7.075(2 \mathrm{H}, \mathrm{d}, J=8.8 \mathrm{~Hz}), 6.949(1 \mathrm{H}, \mathrm{d}$, $J=9.2 \mathrm{~Hz}), 6.865(1 \mathrm{H}, \mathrm{dd}, J=2.8,8.8 \mathrm{~Hz}), 5.103(2 \mathrm{H}, \mathrm{s}), 4.309(2 \mathrm{H}, \mathrm{s})$, $3.846(3 \mathrm{H}, \mathrm{s}), 3.740(3 \mathrm{H}, \mathrm{s}), 3.697(3 \mathrm{H}, \mathrm{s}) ;{ }^{13} \mathrm{C}$ NMR $\delta 165.32,162.45$, 153.67, 152.79, 151.16, 139.01, 131.29, 130.33, 130.05, 128.69, 128.46, 127.62, 127.31, 125.73, 121.38, 115.54, 114.10, 112.83, 112.14, 105.69, 65.55, 58.68, 56.29, 55.92; ESI-MS calculated for $\mathrm{C}_{30} \mathrm{H}_{31} \mathrm{~N}_{2} \mathrm{O}_{7} \mathrm{~S}$ $[\mathrm{M}+\mathrm{H}]^{+}$563.19, found: 562.97 .

4.1.16. $\mathrm{N}$-[3-(2,5-dimethoxy-benzyloxy)-4-(phenylsulfonyl-amino)phenyl]-4-methoxybenzamide (21)

${ }^{1} \mathrm{H}$ NMR $\delta 10.082(1 \mathrm{H}, \mathrm{s}), 9.551(1 \mathrm{H}, \mathrm{s}), 7.925(2 \mathrm{H}, \mathrm{d}, J=8.8 \mathrm{~Hz})$, $7.611(2 \mathrm{H}, \mathrm{m}), 7.473(2 \mathrm{H}, \mathrm{m}), 7.345(3 \mathrm{H}, \mathrm{m}), 7.155(1 \mathrm{H}, \mathrm{d}, J=8.4 \mathrm{~Hz})$, 
$7.099(1 \mathrm{H}, \mathrm{d}, J=3.2 \mathrm{~Hz}), 7.051(2 \mathrm{H}, \mathrm{d}, J=9.2 \mathrm{~Hz}), 6.943(1 \mathrm{H}, \mathrm{d}$, $J=8.8 \mathrm{~Hz}), 6.853(1 \mathrm{H}, \mathrm{dd}, J=3.2,8.8 \mathrm{~Hz}), 4.692(2 \mathrm{H}, \mathrm{s}), 3.833(3 \mathrm{H}, \mathrm{s})$, $3.775(3 \mathrm{H}, \mathrm{s}), 3.758(3 \mathrm{H}, \mathrm{s}) ;{ }^{13} \mathrm{C}$ NMR $\delta 165.30,162.43,153.72$, $152.75,150.48,140.95,139.15,132.73,130.02,129.08,127.41,127.27$, $127.00,125.96,120.82,114.83,114.07,113.12,112.78,111.76,105.65$, $65.14,56.25$, 56.00, 55.89; ESI-MS calculated for $\mathrm{C}_{29} \mathrm{H}_{29} \mathrm{~N}_{2} \mathrm{O}_{7} \mathrm{~S}$ $[\mathrm{M}+\mathrm{H}]^{+}$549.17, found: 548.95 .

\subsubsection{7. $\mathrm{N}$-[3-(2,5-dimethoxy-benzyloxy)-4-(ethanesulfonyl-amino)-} phenyl]-4-iodobenzamide (22)

${ }^{1} \mathrm{H}$ NMR $\delta 10.336(1 \mathrm{H}, \mathrm{s}), 8.944(1 \mathrm{H}, \mathrm{s}), 7.931(2 \mathrm{H}, \mathrm{d}, J=8.8 \mathrm{~Hz})$, $7.744(2 \mathrm{H}, \mathrm{d}, J=8.4 \mathrm{~Hz}), 7.657(1 \mathrm{H}, \mathrm{d}, J=2.4 \mathrm{~Hz}), 7.383(1 \mathrm{H}, \mathrm{dd}, J=2$, $8.4 \mathrm{~Hz}), 7.245(2 \mathrm{H}, \mathrm{m}), 6.982(1 \mathrm{H}, \mathrm{d}, J=8.8 \mathrm{~Hz}), 6.886(1 \mathrm{H}, \mathrm{dd}$, $J=3.2,9.2 \mathrm{~Hz}), 5.063(2 \mathrm{H}, \mathrm{s}), 3.789(3 \mathrm{H}, \mathrm{s}), 3.721(3 \mathrm{H}, \mathrm{s}), 2.962(2 \mathrm{H}$ $\mathrm{q}, J=7.2 \mathrm{~Hz}), 1.131(3 \mathrm{H}, \mathrm{t}, J=7.2 \mathrm{~Hz}) ;{ }^{13} \mathrm{C}$ NMR $\delta 165.22,153.64$, $152.85,151.15,138.62,137.75,134.63,130.05,128.02,125.67,121.69$, $115.56,113.96,112.97,112.10,105.74,99.81,65.52,56.30,55.93$, 46.78, 8.46; ESI-MS calculated for $\mathrm{C}_{24} \mathrm{H}_{26} \mathrm{IN}_{2} \mathrm{O}_{6} \mathrm{~S}[\mathrm{M}+\mathrm{H}]^{+}$: 597.06, found: 596.85 .

4.1.18. $N$-[3-(2,5-dimethoxy-benzyloxy)-4-(propanesulfonyl-

amino)-phenyl]-4-iodobenzamide (23)

${ }^{1} \mathrm{H}$ NMR $\delta 10.335(1 \mathrm{H}, \mathrm{s}), 8.972(1 \mathrm{H}, \mathrm{s}), 7.931(2 \mathrm{H}, \mathrm{d}, J=8.8 \mathrm{~Hz})$, $7.745(2 \mathrm{H}, \mathrm{d}, J=8.8 \mathrm{~Hz}), 7.655(1 \mathrm{H}, \mathrm{d}, J=2.4 \mathrm{~Hz}), 7.389(1 \mathrm{H}, \mathrm{dd}, J=2$, $8.4 \mathrm{~Hz}), 7.262(1 \mathrm{H}, \mathrm{d}, J=3.2 \mathrm{~Hz}), 7.231(1 \mathrm{H}, \mathrm{d}, J=8.4 \mathrm{~Hz}), 6.984(1 \mathrm{H}$, $\mathrm{d}, J=9.2 \mathrm{~Hz}), 6.885(1 \mathrm{H}, \mathrm{dd}, J=3.2,9.2 \mathrm{~Hz}), 5.070(2 \mathrm{H}, \mathrm{s}), 3.793(3 \mathrm{H}$, s), $3.725(3 \mathrm{H}, \mathrm{s}), 2.924(2 \mathrm{H}, \mathrm{m}), 1.655(2 \mathrm{H}, \mathrm{m}), 0.792(3 \mathrm{H}, \mathrm{t}$ $J=7.6 \mathrm{~Hz}) ;{ }^{13} \mathrm{C}$ NMR $\delta 165.22,153.69,152.77,151.06,138.57,137.75$, $134.63,130.05,127.89,125.74,121.77,115.45,113.83,113.00,112.16$, $105.80,99.81,65.46,56.36,55.95,54.14,17.33,13.01$; ESI-MS calculated for $\mathrm{C}_{25} \mathrm{H}_{28} \mathrm{IN}_{2} \mathrm{O}_{6} \mathrm{~S}[\mathrm{M}+\mathrm{H}]^{+} 611.07$, found 610.88 .

4.1.19. N-[3-(2,5-dimethoxy-benzyloxy)-4-(benzylsulfonyl-amino)phenyl]-4-iodobenzamide (24)

${ }^{1} \mathrm{H}$ NMR $\delta 10.349(1 \mathrm{H}, \mathrm{s}), 9.018(1 \mathrm{H}, \mathrm{s}), 7.937(2 \mathrm{H}, \mathrm{d}, J=8.4 \mathrm{~Hz})$, $7.755(2 \mathrm{H}, \mathrm{d}, J=8.4 \mathrm{~Hz}), 7.684(1 \mathrm{H}, \mathrm{d}, J=2 \mathrm{~Hz}), 7.382(1 \mathrm{H}, \mathrm{dd}, J=2$, $8.4 \mathrm{~Hz}), 7.304(6 \mathrm{H}, \mathrm{m}), 7.164(1 \mathrm{H}, \mathrm{d}, J=8.4 \mathrm{~Hz}), 6.948(1 \mathrm{H}, \mathrm{d}$, $J=9.2 \mathrm{~Hz}), 6.864(1 \mathrm{H}, \mathrm{dd}, J=2.8,9.2 \mathrm{~Hz}), 5.104(2 \mathrm{H}, \mathrm{s}), 4.317(2 \mathrm{H}, \mathrm{s})$, $3.738(3 \mathrm{H}, \mathrm{s}), 3.694(3 \mathrm{H}, \mathrm{s}) ;{ }^{13} \mathrm{C}$ NMR $\delta 165.22,153.67,152.71,151.16$, $138.51,137.76,134.65,131.29,130.30,130.05,128.70,128.47,127.50$, 125.68, 121.82, 115.53, 114.07, 112.96, 112.14, 105.81, 99.82, 65.59, 58.70, 56.30, 55.91; ESI-MS calculated for $\mathrm{C}_{29} \mathrm{H}_{28} \mathrm{IN}_{2} \mathrm{O}_{6} \mathrm{~S}[\mathrm{M}+\mathrm{H}]^{+}$ 659.07, found: 658.84 .

4.1.20. $\mathrm{N}$-[3-(2,5-dimethoxy-benzyloxy)-4-(phenylsulfonyl-amino)phenyl]-4-iodobenzamide (25)

${ }^{1} \mathrm{H}$ NMR $\delta 10.278(1 \mathrm{H}, \mathrm{s}), 9.579(1 \mathrm{H}, \mathrm{s}), 7.911(2 \mathrm{H}, \mathrm{d}, J=8.4 \mathrm{~Hz})$, $7.709(2 \mathrm{H}, \mathrm{d}, J=8.8 \mathrm{~Hz}), 7.614(2 \mathrm{H}, \mathrm{m}), 7.464(2 \mathrm{H}, \mathrm{m}), 7.339(3 \mathrm{H}, \mathrm{m})$, $7.173(1 \mathrm{H}, \mathrm{d}, J=8.8 \mathrm{~Hz}), 7.086(1 \mathrm{H}, \mathrm{d}, J=3.2 \mathrm{~Hz}), 6.941(1 \mathrm{H}, \mathrm{d}$, $J=9.2 \mathrm{~Hz}), 6.852(1 \mathrm{H}, \mathrm{dd}, J=3.2,9.2 \mathrm{~Hz}), 4.696(2 \mathrm{H}, \mathrm{s}), 3.770(3 \mathrm{H}, \mathrm{s})$, $3.753(3 \mathrm{H}, \mathrm{s}) ;{ }^{13} \mathrm{C}$ NMR $\delta 165.20,153.71,152.70,150.48,140.92$, $138.68,137.72,134.61,132.76,130.02,129.11,127.35,127.00,125.90$, $121.23,114.82,113.14,112.89,111.78,105.75,99.80,65.17,56.27$, 56.00; ESI-MS calculated for $\mathrm{C}_{28} \mathrm{H}_{26} \mathrm{IN}_{2} \mathrm{O}_{6} \mathrm{~S}[\mathrm{M}+\mathrm{H}]^{+} 645.06$, found 644.83 .

4.1.21. N-[3-(2,5-dimethoxy-benzyloxy)- 4-(ethanesulfonylamino)-phenyl]-3,4-dimethoxybenzamide (26)

${ }^{1} \mathrm{H}$ NMR $\delta 10.129(1 \mathrm{H}, \mathrm{s}), 8.922(1 \mathrm{H}, \mathrm{s}), 7.682(1 \mathrm{H}, \mathrm{d}, J=2.4 \mathrm{~Hz})$, $7.628(1 \mathrm{H}, \mathrm{dd}, J=2.4,8.4 \mathrm{~Hz}), 7.528(1 \mathrm{H}, \mathrm{d}, J=2.4 \mathrm{~Hz}), 7.366(1 \mathrm{H}, \mathrm{dd}$, $J=2,8.4 \mathrm{~Hz}), 7.260(1 \mathrm{H}, \mathrm{d}, J=2.8 \mathrm{~Hz}), 7.227(1 \mathrm{H}, \mathrm{d}, J=8.8 \mathrm{~Hz}), 7.092$ $(1 \mathrm{H}, \mathrm{d}, J=8.4 \mathrm{~Hz}), 6.985(1 \mathrm{H}, \mathrm{d}, J=9.2 \mathrm{~Hz}), 6.889(1 \mathrm{H}, \mathrm{dd}, J=3.2$, $8.8 \mathrm{~Hz}$ ), $5.065(2 \mathrm{H}, \mathrm{s}), 3.847(3 \mathrm{H}, \mathrm{s}), 3.842(3 \mathrm{H}, \mathrm{s}), 3.792(3 \mathrm{H}, \mathrm{s}), 3.727$ $(3 \mathrm{H}, \mathrm{s}), 2.962(2 \mathrm{H}, \mathrm{q}, J=7.2 \mathrm{~Hz}), 1.131(3 \mathrm{H}, \mathrm{t}, J=7.2 \mathrm{~Hz}) ;{ }^{13} \mathrm{C} \mathrm{NMR}$ $\delta 165.36,153.64,152.91,152.22,151.16,148.83,139.03,128.08$,
127.33, 125.70, 121.48, 121.32, 115.61, 113.95, 112.99, 112.08, 111.63, 111.39, 105.73, 65.49, 56.28, 56.17, 56.15, 55.92, 46.76, 8.46; ESI-MS calculated for $\mathrm{C}_{26} \mathrm{H}_{31} \mathrm{~N}_{2} \mathrm{O}_{8} \mathrm{~S}[\mathrm{M}+\mathrm{H}]^{+}$: 531.18, found: 530.97 .

4.1.22. N-[3-(2,5-dimethoxy-benzyloxy)-4-(propanesulfonylamino)-phenyl]-3,4-dimethoxybenzamide (27)

${ }^{1} \mathrm{H}$ NMR $\delta 10.128(1 \mathrm{H}, \mathrm{s}), 8.950(1 \mathrm{H}, \mathrm{s}), 7.678(1 \mathrm{H}, \mathrm{d}, J=2.4 \mathrm{~Hz})$, $7.627(1 \mathrm{H}, \mathrm{dd}, J=2,8.4 \mathrm{~Hz}), 7.526(1 \mathrm{H}, \mathrm{d}, J=2 \mathrm{~Hz}), 7.370(1 \mathrm{H}, \mathrm{dd}$, $J=2,8.8 \mathrm{~Hz}), 7.273(1 \mathrm{H}, \mathrm{d}, J=2.8 \mathrm{~Hz}), 7.223(1 \mathrm{H}, \mathrm{d}, J=8.8 \mathrm{~Hz}), 7.092$ $(1 \mathrm{H}, \mathrm{d}, J=8.8 \mathrm{~Hz}), 6.987$ (1H. d, $J=9.2 \mathrm{~Hz}), 6.888(1 \mathrm{H}, \mathrm{d}, J=2.8$, $9.2 \mathrm{~Hz}), 5.071(2 \mathrm{H}, \mathrm{s}), 3.847(3 \mathrm{H}, \mathrm{s}), 3.842(3 \mathrm{H}, \mathrm{s}), 3.797(3 \mathrm{H}, \mathrm{s}), 3.730$ $(3 \mathrm{H}, \mathrm{s}), 2.922(2 \mathrm{H}, \mathrm{m}), 1.654(2 \mathrm{H}, \mathrm{m}), 0.790(3 \mathrm{H}, \mathrm{t}, J=7.2 \mathrm{~Hz}) ;{ }^{13} \mathrm{C}$ NMR $\delta 165.35,153.70,152.82,152.22,151.07,148.83,138.97,127.94$, $127.33,125.78,121.47,121.41,115.50,113.82,113.02,112.15,111.63$, 111.40, 105.81, 65.44, 56.35, 56.17, 56.15, 55.94, 54.13, 17.34, 13.00; ESI-MS calculated for $\mathrm{C}_{27} \mathrm{H}_{33} \mathrm{~N}_{2} \mathrm{O}_{8} \mathrm{~S}[\mathrm{M}+\mathrm{H}]^{+}$545.20, found: 544.98 .

\subsubsection{N-[3-(2,5-dimethoxy-benzyloxy)-4-(ethanesulfonyl-} amino)-phenyl]-4-bromobenzamide (28)

${ }^{1} \mathrm{H}$ NMR $\delta 10.358(1 \mathrm{H}, \mathrm{s}), 8.947(1 \mathrm{H}, \mathrm{s}), 7.909(2 \mathrm{H}, \mathrm{d}, J=8.8 \mathrm{~Hz})$, $7.760(2 \mathrm{H}, \mathrm{d}, J=8.8 \mathrm{~Hz}), 7.658(1 \mathrm{H}, \mathrm{d}, J=2 \mathrm{~Hz}), 7.384(1 \mathrm{H}, \mathrm{dd}, J=2$, $8.4 \mathrm{~Hz}), 7.248(2 \mathrm{H}, \mathrm{m}), 6.983(1 \mathrm{H}, \mathrm{d}, J=8.8 \mathrm{~Hz}), 6.886(1 \mathrm{H}, \mathrm{dd}$, $J=2.8,8.8 \mathrm{~Hz}), 5.065(2 \mathrm{H}, \mathrm{s}), 3.789(3 \mathrm{H}, \mathrm{s}), 3.721(3 \mathrm{H}, \mathrm{s}), 2.963(2 \mathrm{H}$, $\mathrm{q}, J=7.2 \mathrm{~Hz}), 1.131$ (3H, t, $J=7.2) ;{ }^{13} \mathrm{C}$ NMR $\delta 164.95,153.64,152.85$, $151.15,138.61,134.33,131.88,130.24,128.05,125.89,125.66,121.71$, 115.55, 113.94, 112.97, 112.08, 105.74, 65.51, 56.28, 55.92, 46.78, 8.47; ESI-MS calculated for $\mathrm{C}_{24} \mathrm{H}_{26} \mathrm{BrN}_{2} \mathrm{O}_{6} \mathrm{~S}[\mathrm{M}+\mathrm{H}]^{+}$: 549.07 , found: 548.86 .

4.1.24. N-[3-(2,5-dimethoxy-benzyloxy)-4-(propanesulfonylamino)-phenyl]-4-bromobenzamide (29)

${ }^{1} \mathrm{H}$ NMR $\delta 10.356(1 \mathrm{H}, \mathrm{s}), 8.973(1 \mathrm{H}, \mathrm{s}), 7.909(2 \mathrm{H}, \mathrm{d}, J=8.8 \mathrm{~Hz})$, $7.760(2 \mathrm{H}, \mathrm{d}, J=8.8 \mathrm{~Hz}), 7.652(1 \mathrm{H}, \mathrm{s}), 7.388(1 \mathrm{H}, \mathrm{d}, J=8.4 \mathrm{~Hz}), 7.262$ $(1 \mathrm{H}, \mathrm{d}, J=3.2 \mathrm{~Hz}), 7.234(1 \mathrm{H}, \mathrm{d}, J=8.4 \mathrm{~Hz}), 6.985(1 \mathrm{H}, \mathrm{d}, J=8.8 \mathrm{~Hz})$, $6.885(1 \mathrm{H}, \mathrm{dd}, J=2.8,8.8 \mathrm{~Hz}), 5.071(2 \mathrm{H}, \mathrm{s}), 3.794(3 \mathrm{H}, \mathrm{s}), 3.725(3 \mathrm{H}$, s), $2.924(2 \mathrm{H}, \mathrm{m}), 1.655(2 \mathrm{H}, \mathrm{m}), 0.793(3 \mathrm{H}, \mathrm{t}, J=7.6 \mathrm{~Hz}) ;{ }^{13} \mathrm{C} \mathrm{NMR}$ $\delta 164.95,153.69,152.78,151.04,138.58,134.33,131.88,130.24$, $127.95,125.90,125.73,121.78,115.45,113.77,112.99,112.11,105.78$, $65.44,56.33,55.93,54.13,17.35,13.01$; ESI-MS calculated for $\mathrm{C}_{25} \mathrm{H}_{28} \mathrm{BrN}_{2} \mathrm{O}_{6} \mathrm{~S}[\mathrm{M}+\mathrm{H}]^{+}$563.09, found: 562.91 .

\subsubsection{N-[3-(2,5-dimethoxy-benzyloxy)-4-acetamido-phenyl]-4- methoxybenzamide (30)}

${ }^{1} \mathrm{H}$ NMR $\delta 10.063(1 \mathrm{H}, \mathrm{s}), 9.171(1 \mathrm{H}, \mathrm{s}), 7.950(2 \mathrm{H}, \mathrm{d}, J=8.8 \mathrm{~Hz})$, $7.677(1 \mathrm{H}, \mathrm{d}, J=8.4 \mathrm{~Hz}), 7.623(1 \mathrm{H}, \mathrm{s}), 7.355(1 \mathrm{H}, \mathrm{d}, J=8.8 \mathrm{~Hz}), 7.141$ $(1 \mathrm{H}, \mathrm{d}, J=2.8 \mathrm{~Hz}), 7.058(2 \mathrm{H}, \mathrm{d}, J=8.8 \mathrm{~Hz}), 6.981(1 \mathrm{H}, \mathrm{d}, J=9.2 \mathrm{~Hz})$, $6.859(1 \mathrm{H}, \mathrm{dd}, J=2.8,8.8 \mathrm{~Hz}), 5.097(2 \mathrm{H}, \mathrm{s}), 3.838(3 \mathrm{H}, \mathrm{s}), 3.805(3 \mathrm{H}$, s), $3.718(3 \mathrm{H}, \mathrm{s}), 2.070(3 \mathrm{H}, \mathrm{s}) ;{ }^{13} \mathrm{C}$ NMR $\delta 168.63,165.15,162.36$, $153.68,150.90,149.91,136.92,129.98,127.40,126.16,123.99,123.61$, 114.50, 114.05, 113.66, 112.74, 112.24, 105.88, 65.44, 56.39, 55.90, 55.82, 24.06; ESI-MS calculated for $\mathrm{C}_{25} \mathrm{H}_{27} \mathrm{~N}_{2} \mathrm{O}_{6}[\mathrm{M}+\mathrm{H}]^{+} 451.19$, found: 450.99 .

4.1.26. $N$-[3-(2,5-dimethoxy-benzyloxy)-4-acetamido-phenyl]-3,4dimethoxybenzamide (31)

${ }^{1} \mathrm{H}$ NMR $\delta 10.056(1 \mathrm{H}, \mathrm{s}), 9.172(1 \mathrm{H}, \mathrm{s}), 7.690(1 \mathrm{H}, \mathrm{d}, J=8.8 \mathrm{~Hz})$, $7.606(2 \mathrm{H}, \mathrm{m}), 7.522(1 \mathrm{H}, \mathrm{d}, J=1.6 \mathrm{~Hz}), 7.327(1 \mathrm{H}, \mathrm{d}, J=9.2 \mathrm{~Hz}), 7.142$ $(1 \mathrm{H}, \mathrm{d}, J=3.2 \mathrm{~Hz}), 7.080(1 \mathrm{H}, \mathrm{d}, J=8.4 \mathrm{~Hz}), 6.982(1 \mathrm{H}, \mathrm{d}, J=8.8 \mathrm{~Hz})$, $6.861(1 \mathrm{H}, \mathrm{dd}, J=2.8,8.8 \mathrm{~Hz}), 5.099(2 \mathrm{H}, \mathrm{s}), 3.842(3 \mathrm{H}, \mathrm{s}), 3.837(3 \mathrm{H}$, s), $3.804(3 \mathrm{H}, \mathrm{s}), 3.720(3 \mathrm{H}, \mathrm{s}), 2.072(3 \mathrm{H}, \mathrm{s}) ;{ }^{13} \mathrm{C}$ NMR $\delta 168.65$, $165.21,153.68,152.13,150.91,149.90,148.81,136.85,127.45,126.16$, 123.96, 123.70, 121.42, 114.55, 113.65, 112.92, 112.21, 111.61, 111.38, 106.03, 65.48, 56.36, 56.14, 55.80, 24.07; ESI-MS calculated for $\mathrm{C}_{26} \mathrm{H}_{29} \mathrm{~N}_{2} \mathrm{O}_{7}[\mathrm{M}+\mathrm{H}]^{+}$481.20, found 481.01. 
4.1.27. N-[3-(2,5-dimethoxy-benzyloxy)-4-acetamido-phenyl]-4-

bromobenzamide (32)

${ }^{1} \mathrm{H}$ NMR $\delta 10.286(1 \mathrm{H}, \mathrm{s}), 9.192(1 \mathrm{H}, \mathrm{s}), 7.901(2 \mathrm{H}, \mathrm{d}, J=8.4 \mathrm{~Hz})$, $7.748(2 \mathrm{H}, \mathrm{d}, J=8.4 \mathrm{~Hz}), 7.710(1 \mathrm{H}, \mathrm{d}, J=8.8 \mathrm{~Hz}), 7.600(1 \mathrm{H}, \mathrm{d}$, $J=2 \mathrm{~Hz}), 7.357(1 \mathrm{H}, \mathrm{d}, J=8.4 \mathrm{~Hz}), 7.133(1 \mathrm{H}, \mathrm{d}, J=2.8 \mathrm{~Hz}), 6.981$ $(1 \mathrm{H}, \mathrm{d}, J=9.2 \mathrm{~Hz}), 6.860(1 \mathrm{H}, \mathrm{dd}, J=3.2,8.8 \mathrm{~Hz}), 5.102(2 \mathrm{H}, \mathrm{s}), 3.803$ (3H, s), $3.715(3 \mathrm{H}, \mathrm{s}), 2.075(3 \mathrm{H}, \mathrm{s}) ;{ }^{13} \mathrm{C}$ NMR $\delta 168.65,164.77,153.67$, $150.91,149.85,136.45,134.43,131.84,130.20,126.10,125.76,123.95$, 114.51, 113.67, 112.86, 112.25, 105.96, 99.99, 65.47, 56.39, 55.82, 24.08; ESI-MS calculated for $\mathrm{C}_{24} \mathrm{H}_{24} \mathrm{BrN}_{2} \mathrm{O}_{5}[\mathrm{M}+\mathrm{H}]^{+} 499.09$, found: 498.89 .

4.1.28. $\mathrm{N}$-[3-(2,5-dimethoxy-benzyloxy)-4-acetamido-phenyl]-4iodobenzamide (33)

${ }^{1} \mathrm{H}$ NMR $\delta 10.263(1 \mathrm{H}, \mathrm{s}), 9.188(1 \mathrm{H}, \mathrm{s}), 7.918(2 \mathrm{H}, \mathrm{d}, J=8.8 \mathrm{~Hz})$, $7.730(3 \mathrm{H}, \mathrm{m}), 7.599(1 \mathrm{H}, \mathrm{d}, J=2 \mathrm{~Hz}), 7.253(1 \mathrm{H}, \mathrm{dd}, J=2,8.8 \mathrm{~Hz})$, $7.131(1 \mathrm{H}, \mathrm{d}, J=3.2 \mathrm{~Hz}), 6.979(1 \mathrm{H}, \mathrm{d}, J=8.8 \mathrm{~Hz}), 6.858(1 \mathrm{H}, \mathrm{dd}$, $J=3.2,9.2 \mathrm{~Hz}), 5.099(2 \mathrm{H}, \mathrm{s}), 3.801(3 \mathrm{H}, \mathrm{s}), 3.714(3 \mathrm{H}, \mathrm{s}), 2.073(3 \mathrm{H}$, s); ${ }^{13} \mathrm{C}$ NMR $\delta 168.66,165.04,153.67,150.91,149.86,137.71,136.45$, $134.73,130.02,126.11,123.99,123.95,114.51,113.68,112.86,112.25$, 105.95, 99.66, 65.47, 56.40, 55.82, 24.08; ESI-MS calculated for $\mathrm{C}_{24} \mathrm{H}_{24} \mathrm{IN}_{2} \mathrm{O}_{5}[\mathrm{M}+\mathrm{H}]^{+}$547.07, found 546.86.

\subsection{Biological studies}

\subsubsection{Cell culture}

Four breast cancer cell lines including SKBR-3, MCF-7, MDA-MB231, and MDA-MB-468 were obtained from ATCC (Rockville, MD). The cells were maintained in RPMI1640 medium supplemented with $10 \%$ fetal bovine serum (FBS), $2 \mathrm{mmol} / \mathrm{l}$ L-Glutamine, $1 \mathrm{mmol} / \mathrm{l}$ sodium pyruvate, $100 \mathrm{U} / \mathrm{ml}$ penicillin-streptomycin. FBS was heat inactivated for $30 \mathrm{~min}$ in a $56^{\circ} \mathrm{C}$ water bath before use. Cell cultures were grown at $37{ }^{\circ} \mathrm{C}$, in a humidified atmosphere of $5 \% \mathrm{CO}_{2}$ in a VWR $\mathrm{CO}_{2}$ incubator (Bridgeport $\mathrm{NJ}$ ).

\subsubsection{Cell viability analysis}

The effects of the new derivatives on the viability of four breast cancer cell lines were assessed using the 3-(4,5-dimethylthiazol-2yl)-2,5-diphenyl-2H-tetrazolium bromide assay in six replicates. Cells were grown in RPMI1640 medium in 96-well, flat-bottomed plates for $24 \mathrm{~h}$, and were exposed to various concentrations of the compounds dissolved in DMSO (final concentration $0.1 \%$ ) in media for $48 \mathrm{~h}$. Controls received DMSO vehicle at a concentration equal to that in drug-treated cells. The medium was removed, replaced by $200 \mu \mathrm{L}$ of $0.5 \mathrm{mg} / \mathrm{ml}$ of 3-(4,5-dimethylthiazol-2-yl)2,5-diphenyl-2H-tetrazolium bromide in fresh media, and cells were incubated in the $\mathrm{CO}_{2}$ incubator at $37^{\circ} \mathrm{C}$ for $2 \mathrm{~h}$. Supernatants were removed from the wells, and the reduced 3-(4,5dimethylthiazol-2-yl)-2,5-diphenyl-2H-tetrazolium bromide dye was solubilized in $200 \mu \mathrm{L} /$ well DMSO. Absorbance at $570 \mathrm{~nm}$ was determined on a plate reader. Statistical and graphical information was determined using GraphPad Prism software (GraphPad Software Incorporated) and Microsoft Excel (Microsoft Corporation). $\mathrm{IC}_{50}$ values were determined using nonlinear regression analysis.

\subsubsection{Tubulin polymerization assay}

$100 \mu \mathrm{L}$ microtubule-associated protein-rich tubulin $(2 \mathrm{mg} / \mathrm{ml}$, bovine brain, Cytoskeleton) in buffer containing $80 \mathrm{mM}$ PIPES ( $\mathrm{pH}$ 6.9), $2 \mathrm{mM} \mathrm{MgCl}, 0.5 \mathrm{mM}$ EGTA, and 5\% glycerol was mixed with DMSO (as control) or various concentrations of $\mathbf{1 0}$ and $\mathbf{1 2}$ in DMSO and incubated at $37{ }^{\circ} \mathrm{C} .1 \mu \mathrm{L}$ of $100 \mathrm{mM}$ GTP was added to the mixture to initiate the tubulin polymerization. The absorbance was monitored at $340 \mathrm{~nm}$ over 20 min using a Varian Cary 50 series spectrophotometer.

\subsubsection{Hsp27 chaperone activity assay}

A mixture of $0.24 \mathrm{mg} / \mathrm{ml}$ human recombinant insulin (Life Technologies), $0.02 \mathrm{mg} / \mathrm{ml} \mathrm{Hsp27} \mathrm{(Cell} \mathrm{Sciences),} \mathrm{and} \mathrm{compounds}$ 10 and 12 in $98 \mu \mathrm{l}$ sodium phosphate buffer, $\mathrm{pH}$ 7.4, was incubated at $37{ }^{\circ} \mathrm{C}$ for $5 \mathrm{~min}$, whereupon $2 \mu \mathrm{L}$ of $1 \mathrm{M}$ DTT in the same assay buffer was added to initiate the insulin aggregation. The absorbance was monitored at $400 \mathrm{~nm}$ over $30 \mathrm{~min}$ using a Varian Cary 50 series spectrophotometer. A mixture of $0.24 \mathrm{mg} / \mathrm{ml}$ insulin in the absence or presence of $0.02 \mathrm{mg} / \mathrm{ml} \mathrm{Hsp27}$ with DMSO was used as control.

\section{Molecular docking simulation}

Molecular docking of compounds $\mathbf{1 0}$ and $\mathbf{1 2}$ into the crystal structure of tubulin was performed using software AUTODOCK 4. The X-ray crystal structures of tubulin with PDB code 1SA0 from Protein Data Bank was used for docking simulation of compounds into the colchicine binding site. To prepare tubulin for autodock, all hydrogens were added and the ligand with identifier CN2700 in 1SA0 was deleted. Compound $\mathbf{1 0}$ and $\mathbf{1 2}$ were drawn and energy minimized with MM2 force field using Chem3D Ultra 10.0 (Cambridge Soft Corp., US). Autogrid was used to pre-calculate the grid maps of the binding energy between tubulin and compounds. A grid box size of $44 \times 46 \times 42$ points in $x, y$ and $z$ directions was built and the grid center was located in $x=116.909, y=89.688$, $z=7.094$. For running autodock, Lamarckian genetic algorithm was chosen as the search method. Genetic algorithm parameters were set as default. The top docked conformation for each compound was retained for analysis.

\section{Acknowledgments}

This research was supported by a startup fund from Cleveland State University, and also partially supported by grant R15AI 103889 (B. Su), Center for Gene Regulation in Health and Disease (GRHD) of Cleveland State University and Ohio Department of Development (ODOD) and National Science Foundation Major Research Instrumentation Grants (CHE-0923398 and CHE1126384).

\section{Abbreviations}

\section{Hsp27 heat shock protein 27}

SAR structure-activity relationship

Her2 human epidermal growth factor receptor 2

FBS fetal bovine serum

DTT dithiothreitol

\section{Appendix A. Supplementary data}

Supplementary data related to this article can be found at http:// dx.doi.org/10.1016/j.ejmech.2014.04.038.

\section{References}

[1] H.J. Arts, H. Hollema, W. Lemstra, P.H. Willemse, E.G. De Vries, H.H. Kampinga A.G. Van der Zee, Heat-shock-protein-27 (hsp27) expression in ovarian carcinoma: relation in response to chemotherapy and prognosis, International Journal of Cancer 84 (1999) 234-238.

[2] C. Garrido, P. Mehlen, A. Fromentin, A. Hammann, M. Assem, A.P. Arrigo, B. Chauffert, Inconstant association between $27-\mathrm{kDa}$ heat-shock protein (Hsp27) content and doxorubicin resistance in human colon cancer cells. The doxorubicin-protecting effect of Hsp27, European Journal of Biochemistry 237 (1996) 653-659.

[3] H.S. Hsu, J.H. Lin, W.C. Huang, T.W. Hsu, K. Su, S.H. Chiou, Y.T. Tsai, S.C. Hung, Chemoresistance of lung cancer stemlike cells depends on activation of Hsp27, Cancer 117 (2011) 1516-1528. 
[4] C. Garrido, M. Brunet, C. Didelot, Y. Zermati, E. Schmitt, G. Kroemer, Heat shock proteins 27 and 70: anti-apoptotic proteins with tumorigenic properties, Cell Cycle 5 (2006) 2592-2601.

[5] M. Kamada, A. So, M. Muramaki, P. Rocchi, E. Beraldi, M. Gleave, Hsp27 knockdown using nucleotide-based therapies inhibit tumor growth and enhance chemotherapy in human bladder cancer cells, Molecular Cancer Therapeutics 6 (2007) 299-308.

[6] C. Garrido, E. Solary, A role of HSPs in apoptosis through "protein triage"? Cell Death and Differentiation 10 (2003) 619-620.

[7] C. Garrido, J.M. Bruey, A. Fromentin, A. Hammann, A.P. Arrigo, E. Solary, HSP27 inhibits cytochrome c-dependent activation of procaspase-9, FASEB Journal 13 (1999) 2061-2070.

[8] C.G. Concannon, A.M. Gorman, A. Samali, On the role of Hsp27 in regulating apoptosis, Apoptosis 8 (2003) 61-70.

[9] V. Baylot, C. Andrieu, M. Katsogiannou, D. Taieb, S. Garcia, S. Giusiano, J. Acunzo, J. Iovanna, M. Gleave, C. Garrido, P. Rocchi, OGX-427 inhibits tumor progression and enhances gemcitabine chemotherapy in pancreatic cancer, Cell Death \& Disease 2 (2011) e221.

[10] G.O. Elpek, S. Karaveli, T. Simsek, N. Keles, N.H. Aksoy, Expression of heatshock proteins hsp27, hsp70 and hsp90 in malignant epithelial tumour of the ovaries, APMIS 111 (2003) 523-530.

[11] S.H. Kang, K.W. Kang, K.H. Kim, B. Kwon, S.K. Kim, H.Y. Lee, S.Y. Kong, E.S. Lee S.G. Jang, B.C. Yoo, Upregulated HSP27 in human breast cancer cells reduces Herceptin susceptibility by increasing Her2 protein stability, BMC Cancer 8 (2008) 286-2407, 8-286.

[12] R. Langer, K. Ott, K. Specht, K. Becker, F. Lordick, M. Burian, K. Herrmann, A. Schrattenholz, M.A. Cahill, M. Schwaiger, H. Hofler, H.J. Wester, Protein expression profiling in esophageal adenocarcinoma patients indicates association of heat-shock protein 27 expression and chemotherapy response Clinical Cancer Research 14 (2008) 8279-8287.

[13] M. Sherman, G. Multhoff, Heat shock proteins in cancer, Annals of the New York Academy of Sciences 1113 (2007) 192-201.

[14] J.C. Heinrich, A. Tuukkanen, M. Schroeder, T. Fahrig, R. Fahrig, RP101 (brivudine) binds to heat shock protein HSP27 (HSPB1) and enhances survival in animals and pancreatic cancer patients, Journal of Cancer Research and Clinical Oncology 137 (2011) 1349-1361.

[15] D. Chauhan, G. Li, R. Shringarpure, K. Podar, Y. Ohtake, T. Hideshima, K.C. Anderson, Blockade of Hsp27 overcomes bortezomib/proteasome inhibitor PS-341 resistance in lymphoma cells, Cancer Research 63 (2003) 61746177.

[16] R. Heald, E. Nogales, Microtubule dynamics, Journal of Cell Science 115 (2002) 3-4.

[17] H.J. Ahn, Y.S. Kim, J.U. Kim, S.M. Han, J.W. Shin, H.O. Yang, Mechanism of taxolinduced apoptosis in human SKOV3 ovarian carcinoma cells, Journal of Cellular Biochemistry 91 (2004) 1043-1052.

[18] G.C. Das, D. Holiday, R. Gallardo, C. Haas, Taxol-induced cell cycle arrest and apoptosis: dose-response relationship in lung cancer cells of different wildtype p53 status and under isogenic condition, Cancer Letters 165 (2001) $147-153$.

[19] V.M. Pushkarev, D.V. Starenki, V.A. Saenko, H. Namba, J. Kurebayashi, M.D. Tronko, S. Yamashita, Molecular mechanisms of the effects of low concentrations of taxol in anaplastic thyroid cancer cells, Endocrinology 145 (2004) 3143-3152.

[20] V.M. Pushkarev, D.V. Starenki, V.A. Saenko, S. Yamashita, O.I. Kovzun, I.D. Popadiuk, V.V. Pushkarev, M.D. Tronko, Effects of low and high concentrations of antitumour drug taxol in anaplastic thyroid cancer cells, Experimental Oncology 31 (2009) 16-21.

[21] Y. Matsui, B.A. Hadaschik, L. Fazli, R.J. Andersen, M.E. Gleave, A.I. So, Intravesical combination treatment with antisense oligonucleotides targeting heat shock protein-27 and HTI-286 as a novel strategy for high-grade bladder cancer, Molecular Cancer Therapeutics 8 (2009) 2402-2411.

[22] B. Zhong, S. Chennamaneni, R. Lama, X. Yi, W.J. Geldenhuys, J.J. Pink, A. Dowlati, Y. Xu, A. Zhou, B. Su, Synthesis and anticancer mechanism investigation of dual Hsp27 and tubulin inhibitors, Journal of Medicinal Chemistry 56 (2013) 5306-5320.

[23] X. Yi, B. Zhong, K.M. Smith, W.J. Geldenhuys, Y. Feng, J.J. Pink, A. Dowlati, Y. Xu, A. Zhou, B. Su, Identification of a class of novel tubulin inhibitors, Journal of Medicinal Chemistry 55 (2012) 3425-3435. 